Pages

L. W. Szczerba and A. Tarski, Metamathematical discussion of some affine geometries 155-192 A. Prestel and L. W. Szczerba, Non-axiomatizability of real general affine geometry 193-202 D. E. Marshall and A. G. O'Farrell, Uniform approximation by real functions. . . 203-211 G. L. Cherlin, Lindenbaum algebras and model companions . . . . . . . . . 213-219 M. Smith and S. Young, Periodic homeomorphisms on T-like continua . . . . . 221-224

P. Strantzalos, Eigentlich operierende Gruppen von Isometrien . . . . . . . . 225-232

Les FUNDAMENTA MATHEMATICAE publient, en langues des congrès internationaux, des travaux consacrés à la Théorie des Ensembles, Topologie, Fondements de Mathématiques, Fonctions Réelles, Théorie Descriptive des Ensembles, Algèbre Abstraite

Chaque volume paraît en 3 fascicules

Adresse de la Rédaction:

FUNDAMENTA MATHEMATICAE, Śniadeckich 8, 00-950 Warszawa (Pologne)

Adresse de l'Échange:

INSTITUT MATHÉMATIQUE, ACADÉMIE POLONAISE DES SCIENCES Sniadeckich 8, 00-950 Warszawa (Pologne)

Tous les volumes sont à obtenir par l'intermédiaire de

ARS POLONA, Krakowskie Przedmieście 7, 00-068 Warszawa (Pologne)

Correspondence concerning editorial work and manuscripts should be addressed to: FUNDAMENTA MATHEMATICAE, Sniadeckich 8, 00-950 Warszawa (Poland)

Correspondence concerning exchange should be addressed to: INSTITUTE OF MATHEMATICS, POLISH ACADEMY OF SCIENCES, Exchange, Sniadeckich 8, 00-950 Warszawa (Poland)

The Fundamenta Mathematicae are available at your bookseller or at ARS POLONA, Krakowskie Przedniescie 7, 00-068 Warszawa (Poland)

(C) Copyright by Państwowe Wydawnicto Naukowe, Warszawa 1979

ISBN 83-01-01395-8～ISSN 0016-2736

DRUKARNIA UNIWERSYTETU JAGIELLOŃSKIEGOW KRAKOWIE

\section{Metamathematical discussion of some affine geometries}

by

L. W. Szczerba (Warszawa) and A. Tarski (Berkeley, Cal.)

Abstract. If in the usual axiom system, of elementary Euclidean geometry we omit Euclid's axiom, we obtain the elementary absolute geometry. If we apply the same procedure to an elementary affine geometry we shall arrive at a much more general geometry (called the elementary general affine geometry). This geometry exhibits a great variety of models. Metamathematical properties of elementary general affine geometry, and some its extensions, are studied.

\section{Contents}

\$1. Preliminaries. Definition of general affine geometry $\mathbf{G A}_{2} \ldots \ldots \ldots 157$ \$2. Construction of an ordered real-closed field . . . . . . . . . . . . . . . . . . . 161 \$3. Representation problens . . . . . . . . . . . . . . . . . . 168 $\S 4$. Metamathematical properties of $\mathrm{GA}_{2} \ldots \ldots \ldots \ldots \ldots$ \$ 5. Uniformly definable extensions of $\mathbf{G A}_{2} \ldots \ldots \ldots \ldots \ldots \ldots$ 8. Examples of extending $G A_{2}$. \$7. Multi-dimensional geometries $\mathbf{G A}_{n} \ldots \ldots \ldots \ldots$

This paper contains a full exposition of joint results of the two authors presented by Tarski at the International Congress for Logic, Methodology and Philosophy of Science (Jerusalem 1964), and subsequently announced in print in Szczerba and Tarski [13]. It is closely related to two earlier publications, Tarski [16] and Szmielew [14]. In those articles certain geometrical theories are described and some of their basic metamathematical properties are established.

The main subject of Tarski [16] is elementary n-dimensional metric Euclidean geometry $\mathbf{E}_{n}$, where $n$ is an arbitrary integer $\geqslant 2$. In Szmielew [14] two related theories are discussed, the elementary n-dimensional metric hyperbolic geometry $\mathbf{H}_{n}$, known also as Bolyai-Lobacevskian geometry, and the elementary n-dimensional metric absolute geometry $\mathbf{A}_{n}$. When referring to these theories we shall usually omit the words "elementary" and "metric". The geometries $\mathbf{E}_{n}, \mathbf{H}_{n}, \mathbf{A}_{n}$, and indeed any elementary metric geometries are treated as theories formalized within first order predicate logic with just two non-logical constants (primitive notions): the ternary predicate $\boldsymbol{B}$ denoting the betweenness relation between three points, and the quaternary relation $D$ denoting the equidistance relation between two pairs of points.

Axioms for $\mathbf{E}_{n}$ are obtained by modifying an axiom system which provides an adeq uate basis for the ordinary (non-elementary) $n$-dimensional Euclidean geometry 1 - Fundamenta Mathematicae $\mathrm{CV} / \mathrm{s}$ 
The modification consists in replacing the continuity axiom (which, as is well-known, cannot be expressed in first-order logic) by an infinite collection of axioms falling under the so-called continuity schema - loosely speaking, by all the first-order instances of the continuity axiom. The method of obtaining an axiomatization for $\mathbf{H}_{n}$ could be described analogously by referring to the ordinary hyperbolic geometry. We can say more simply, however, that an axiom system for $\mathbf{H}_{n}$ is obtained from that of $\mathbf{E}_{n}$ by replacing Euclid's axiom by its negation.

If Euclid's axiom is omitted altogether, we arrive at the axiom system of $\mathbf{A}$ The main results established in Szmielew [14] and T'arski [16] for these theories are the following: the class of all models of each theory is characterized in algebraic terms; $\mathbf{E}_{n}$ and $\mathbf{H}_{n}$ are shown to be complete and consistent, while $\mathbf{A}_{n}$ proves to be incomplete, and indeed to have just two complete and consistent extensions, $\mathbf{E}_{n}$ and $\mathbf{H}_{n}$; each of the theories discussed is decidable, and none of them is finitely" axiomatizable.

In this paper we discuss analogous problems concerning certain affine geometres closely related to $\mathbf{E}_{n}, \mathbf{H}_{n}$, and $\mathbf{A}_{n}$. Just as in Szmielew [14] and Tarski [16], the detailed discussion will be restricted to the case $n=2$. In the last section we shall indicate how our result can be extended to higher dimensions.

With any metric geometry $\mathbf{G}$ formalized in the manner previously indicated we associate the affine geometry AG determined by the following stipulations: the language of $\mathbf{A G}$ is obtained from that of $\mathbf{G}$ by eliminating the symbol $D$, so that $B$ remains as the only non-logical constant; a sentence in the language of $\mathbf{A G}$ is a theorem of $\mathbf{A G}$ iff it is a theorem of $\mathbf{G}$. This approach to affine geometry is known from the literature; see e.g. Whitehead [19] (where the term "descriptive" instead. of "affine" is used). It is essentially equivalent to the more familiar approach that consists in constructing affine geometry as projective geometry in which a certain line, the line "at infinity", has been singled out.

We shall be interested in particular in the affine geometries $\mathbf{A E} \mathbf{E}_{2}, \mathbf{A H}$, and $\mathbf{A A _ { 2 }}$. The way in which these theories have been defined does not yield directly suitable axiom systems for them. In the case of $\mathbf{A E}_{2}$, however, we easily obtain an adequate axiomatization by proceding exactly as in the metric case, i.e., by replacing, in a suitable axiom system of non-elementary affine geometry, the axiom of continuity by the set of all its first-order instances. To obtain an axiomatization for $\mathbf{A H}_{2}$ and $\mathbf{A A}_{2}$ we could try to follow the simple procedure applied in the metric case, namely to modify the axiom system for $\mathbf{A E}_{2}$ by negating Euclid's axiom or by omitting this axiom altogether. It turns out, however, that this method fails, at least when applied to the special axiom system for $\mathbf{A E}_{2}$ which will be adopted in the present paper. Axiomatic theories obtained by this method prove to be proper subtheories of $\mathbf{A H} \mathbf{H}_{2}$ and $\mathbf{A} \mathbf{A}_{2}$. Nevertheless, these theories deserve interest in their own right; this applies especjally to the theory obtained by deleting Euclid's axiom from the axiom system of $\mathbf{A A}_{2}$. The resulting theory, which will be referred to as general affine geometry with continuity schema, or simply general affine geometry, and denoted by $\mathbf{G A}_{2}$, distinguishes itself by the simple form and clear mathematical content of its axicms and by a great variety of interesting extensions. The study of metamathematical properties of $\mathbf{G A}_{2}$ and its extensions is the main topic of this paper.

Since the first report on the results presented in this paper there have been some new developments in the metamathematical study of affine geometries which will be referred to and partially applied in the subsequent discussion. In particular, Szczerba has studied the models of a theory which is more general (and hence weaker) than $\mathbf{G A}_{2}$ and which should properly be called general affine geometry without continuity schema. The main results of his study have been embodied in his doctoral dissertation Szczerba [9], and reported (with short outlines of proofs) in Szczerba [11]. In this connection we have noticed that it is possible to simplify and shorten a part of our paper (namely, the part devoted to the discussion of the representation problem for $\mathbf{G A}_{2}$ in Sections 2 and 3) if we disregard our original proofs, and use instead, as far as it is feasible, the results of Szczerba. We have indeed decided to follow this way (realizing that as a consequence our present paper will not be self-contained). Although the development in Szczerba [9] is much more detailed than in Szczerba [11], we shall refer mainly to the latter paper, since it is more accessible to the reader.

We take this opportunity to express our sincere gratitude to Dr. Steven Givant for his help in preparing this paper for publication.

$\S 1$. Preliminaries. Definition of general affine geometry $\mathbf{G A}_{2}$. In logical and metamathematical notation we shall adhere, in general, to Henkin-Monk-Tarski [1] introducing, however, a few changes. All the expressions written in logical symbols, are treated not as expressions of a formal language but as metamathematical designations of such expressions. As the symbols of quantifiers we shall use $\forall$ and $\exists$. The set of formulas expressed in terms of the ternary relation $\boldsymbol{B}$ will be denoted by $\mathbf{L}(\boldsymbol{B})$; similarly the set of all formulas expressed in terms of the constants $\mathbf{0}, \mathbf{1}$, the operations,$+ \cdot$, and relation $\leqslant$ will be denoted by $\mathbf{L}(\mathbf{0}, \mathbf{1},+, \cdot, \leqslant)\left({ }^{1}\right)$.

We shall consistently employ bold italics (and other bold types) as metamathematical constants to denote variables, individual constants, predicates, and operation symbols occurring in a formal theory. In particular we assume that all the variables occurring in a formal language have been arranged in an infinite sequence (without repetitions) $\left\langle v_{0}, \ldots, v_{n}, \ldots\right\rangle$. To simplify a little the notation we introduce the abbreviations $a=v_{0}, b=v_{1}$, and so on (till the end of the alphabet). Similarly $a^{\prime}=v_{26}, \ldots$ and $a^{\prime \prime}=v_{52}, \ldots$ etc. The corresponding lightface types will be used as variables ranging over elements of relational structures, the relations between such elements, and operations on such elements. We shall use symbols $\mathbf{v}_{0}, \mathbf{v}_{1}, \ldots$ and $\mathbf{v}_{0}^{\prime}, \mathbf{v}_{1}^{\prime}, \ldots$ as metamathematical variables ranging over variables of a formal language, and symbols $\Phi, \Psi, \Gamma$ and $\Delta$ as variables ranging over formulas. of formal languages.

(1) Symbols $+, \cdot, \leqslant$ in $\mathbf{L}(\mathbf{0}, \mathbf{1},+, \cdot, \leqslant)$ should be printed boldface. But for technical reasons here and below will be printed lightface. We hope it will create no misunderstanding. 
By a universal formula we mean any formula of the form $\forall \mathbf{v}_{1} \ldots \forall \mathbf{v}_{n} \Phi$ where $n=0,1, \ldots$, and $\Phi$ is a quantifier-free formula. Similarly, by a $\forall \exists$-formula we mean any formula of the form

$$
\forall \mathbf{v}_{1} \ldots \forall \mathbf{v}_{n} \exists \mathbf{v}_{1}^{\prime} \ldots \exists \mathbf{v}_{m}^{\prime} \Phi
$$

where $n, m=0,1, \ldots$ and $\Phi$ is a quantifier-free formula. We say that a theory. $\mathbf{T}$ is a $\forall \exists$-theory if there is a set of $\forall \exists$-sentences $\mathbf{S}$ such that $\mathbf{T}$ is the set of logical consequences of $\mathbf{S}$; in other words, $\boldsymbol{S}$ is an axiom system of $\mathbf{T}$.

The result of simultaneous substitution of $n$ variables $\mathbf{v}_{0}^{\prime}, \ldots, \mathbf{v}_{n}^{\prime}$ for $n$ distinct variables $\mathbf{v}_{0}, \ldots, \mathbf{v}_{n}$ in the formula $\Phi$ will be denoted by

$$
\Phi\left(\begin{array}{l}
\mathbf{v}_{0}, \ldots, \mathbf{v}_{n} \\
\mathbf{v}_{0}^{\prime}, \ldots, \mathbf{v}_{n}^{\prime}
\end{array}\right) .
$$

In some special, frequently occurring cases we introduce simplified notation: instead of $\Phi\left(\begin{array}{c}\boldsymbol{x} \\ \mathbf{v}_{0}\end{array}\right)$ we shall write $\Phi\left(\mathbf{v}_{0}\right)$, instead of $\Phi\left(\begin{array}{ll}\boldsymbol{x} & y \\ \mathbf{v}_{0} & \mathbf{v}_{1}\end{array}\right)-\Phi\left(\mathbf{v}_{0} \mathbf{v}_{1}\right)$, instead

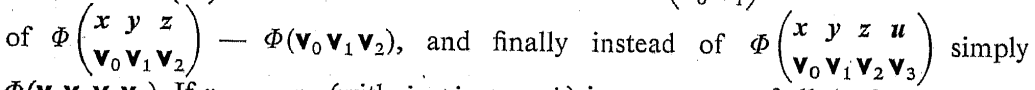
$\Phi\left(\mathbf{v}_{0} \mathbf{v}_{1} \mathbf{v}_{2} \mathbf{v}_{3}\right)$. If $\boldsymbol{v}_{i_{0}}, \ldots, \boldsymbol{v}_{i_{n}}$ (with $i_{0}<i_{1}<\ldots<i_{n}$ ) is a sequence of all the free variables of formula $\Phi$, then by $\llbracket \Phi \rrbracket$, the closure of $\Phi$, we shall mean the sentence

$$
\forall v_{i_{0}} \ldots \forall v_{i_{n}} \Phi \text {. }
$$

Given any formula $\Phi$ in which no variables different from the pairwise distinct $\mathbf{v}_{0}, \ldots, \mathbf{v}_{n}$ occur free, the meaning of the phrase " $\Phi$ is satisfied in the relational structure $\mathfrak{Q}$ when variables $\mathbf{v}_{0}, \ldots, \mathbf{v}_{n}$ are correlated with elements $v_{0}, \ldots, v_{n}$ of $\mathfrak{A}$ respectively", in symbols

$$
\mathfrak{N} \vDash \Phi\left[\begin{array}{ccc}
\mathbf{v}_{0} & \ldots & \mathbf{v}_{n} \\
v_{0} & \ldots & v_{n}
\end{array}\right],
$$

is assumed to be understood. We also assume that it is known under what conditions a relational structure $\mathfrak{V}$ is a model of a sentence $\Phi$, or a set of sentences $S$, or a theory $T$ (in symbols: $\mathfrak{A} \vDash \Phi, \mathfrak{A} \vDash S$ and $\mathfrak{A} \vDash \mathbf{T}$ ), respectively. Given any relational structure $\mathfrak{N}$, by the elementary theory of $\mathfrak{N}$, in symbols $\mathbf{T}(\mathfrak{Q})$, we understand the set of all sentences having $\mathfrak{A}$ as a model; the elementary theory $\mathbf{T}(\mathscr{A})$ of a class $\mathscr{A}$ of relational structures is the intersection of all theories $\mathbf{T}(\mathfrak{I})$ with $\mathfrak{Q} \in \mathscr{A}$.

The models of the formulas in $\mathbf{L}(\mathbf{0}, \mathbf{1},+, \cdot, \leqslant)$ are algebraic structures of the form $\mathfrak{\xi}=\langle F, 0,1,+, \cdot, \leqslant\rangle$ where 0 , and 1 are elements of $F,+$ and $\cdot$ are binary operations on $F$, and $\leqslant$ is a binary relation on $F$. We shall refer to such algebraic structures simply as algebras.

When speaking of extensions of a theory $\mathbf{T}$ formalized in the language of affine geometry (i.e., $\mathbf{T} \subseteq \mathbf{L}(\boldsymbol{B})$ ) we shall refer exclusively to theories formalized within the same language. The models of such theories are the relational structures $\mathfrak{A}=\langle A, B\rangle$ where $A$ is a non-empty set and $B$ a ternary relation between elements of $A$, i.e., a subset of the Cartesian cube $A \times A \times A$.

We say that three elements $a, b, c$ are collinear in $\mathfrak{N}$ (in symbols $L(a b c)$ ) if $B(a b c)$ or $B(b c a)$ or $B(c a b)$ holds. By the line passing through elements $a$ and $b$ we mean the set

$$
L(a b)=\{c: L(a b c)\} .
$$

Note that if $a=b$ then $L(a b)=A$. We shall also use the notions of closed segment $[a c]=\{b: B(a b c)\}$,

open segment,

and right-hand open segment,

$$
(a c)=[a c] \backslash\{a, c\}
$$

$[a c)=[a l c] \backslash\{c\}$

DeFINITION 1.1. By general affine two-dimensional geometry with continuity schema, or briefly general affine geometry (in symbols $\mathbf{G A}_{2}$ ) we mean the (first order) theory based upon the following axioms $\mathbf{A}_{1}-\mathbf{A}_{8}, \mathbf{A}_{9}^{\phi \Psi}$ :

$\mathbf{A}_{1}=\llbracket \boldsymbol{B}(\boldsymbol{a} \boldsymbol{b} \boldsymbol{a}) \rightarrow \boldsymbol{a}=\boldsymbol{b} \rrbracket \quad$ Identity axiom,

$\mathbf{A}_{2}=\llbracket \boldsymbol{B}(\boldsymbol{a b c}) \wedge \boldsymbol{B}(\boldsymbol{b c d}) \wedge \neg \boldsymbol{b}=\boldsymbol{c} \rightarrow \boldsymbol{B}(\boldsymbol{a b d}) \rrbracket \quad$ Transitivity axiom,

$\mathbf{A}_{3}=\llbracket \boldsymbol{B}(\boldsymbol{a} b \boldsymbol{c}) \wedge \boldsymbol{B}(\boldsymbol{a b c}) \wedge \neg \boldsymbol{a}=\boldsymbol{b} \rightarrow \boldsymbol{B}\left(\boldsymbol{a c c} \boldsymbol{c}^{\prime}\right) \vee \boldsymbol{B}\left(\boldsymbol{a c ^ { \prime }} \boldsymbol{c}\right) \rrbracket$ Connectivity axiom,

$\mathbf{A}_{4}=\llbracket \exists a B(a b c) \wedge \neg a=b \rrbracket \quad$ Extension axiom,

$\mathbf{A}_{5}=\llbracket \boldsymbol{B}\left(\boldsymbol{a} \boldsymbol{b}^{\prime} \boldsymbol{c}\right) \wedge \boldsymbol{B}\left(\boldsymbol{b} \boldsymbol{c}^{\prime} \boldsymbol{b}^{\prime}\right) \rightarrow \exists \boldsymbol{a}^{\prime}\left[\boldsymbol{B}\left(\boldsymbol{c} \boldsymbol{a}^{\prime} \boldsymbol{b}\right) \wedge \boldsymbol{B}\left(\boldsymbol{a} \boldsymbol{c}^{\prime} \boldsymbol{a}^{\prime}\right)\right] \rrbracket$ Pasch's axiom (see

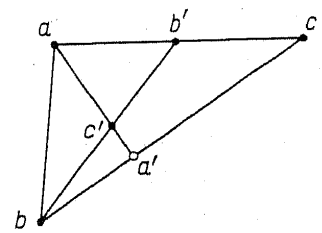

Fig. 1.2),

Fig. 1.2

$\mathbf{A}_{6}=\llbracket B\left(p a a^{\prime}\right) \wedge B\left(p b b^{\prime}\right) \wedge B\left(p c c^{\prime}\right) \wedge B\left(a b c^{\prime \prime}\right) \wedge B\left(a^{\prime} b^{\prime} c^{\prime \prime}\right) \wedge B\left(b c a^{\prime \prime}\right) \wedge$ $\wedge \boldsymbol{B}\left(\boldsymbol{b}^{\prime} \boldsymbol{c}^{\prime} \boldsymbol{a}^{\prime \prime}\right) \wedge \boldsymbol{B}\left(\boldsymbol{a c} \boldsymbol{b}^{\prime \prime}\right) \wedge \boldsymbol{B}\left(\boldsymbol{a}^{\prime} \boldsymbol{c}^{\prime} \boldsymbol{b}^{\prime \prime}\right) \wedge \neg \boldsymbol{B}(\boldsymbol{p} a b) \wedge \neg B(a b p) \wedge$ $\wedge \neg B(b p a) \wedge \neg B(p b c) \wedge \neg B(b c p) \wedge \neg B(c p b) \wedge \neg B(p c a) \wedge$ $\wedge \neg B($ cap $\left.) \wedge \neg B(a p c) \rightarrow B\left(a^{\prime \prime} b^{\prime \prime} c^{\prime \prime}\right)\right] \quad$ Desargues' axiom (see Fig. 1.3)

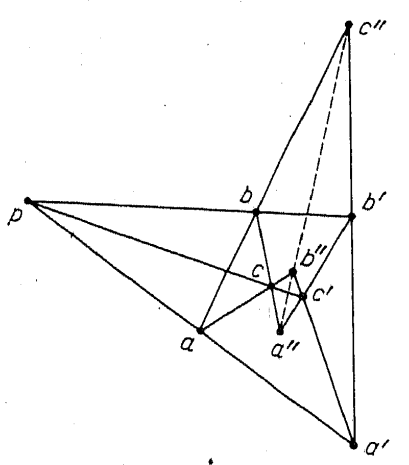

Fig. 1.3 
$\mathbf{A}_{7}=\llbracket \exists a b c[\neg \boldsymbol{B}(\boldsymbol{a b c}) \wedge \neg \boldsymbol{B}(\boldsymbol{b c a}) \wedge \neg \boldsymbol{B}(\boldsymbol{c a b})] \rrbracket$ Lower dimension axiom,

$\mathbf{A}_{8}=\llbracket \exists q[\{B(b q c) \wedge[B(a q p) \vee B(q p a) \vee B(p a q)]\} \vee$

$\vee\{B(c q a) \wedge[B(b q p) \vee B(q p b) \vee B(p b q)]\} \vee$

$\vee\{B(a q \dot{b}) \wedge[B(c q p) \vee B(q p c) \vee B(p c q)]\}]]$

Upper dimension axiom (see Fig. 1.4),

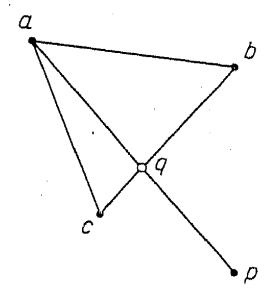

Fig. 1.4

$\mathbf{A}_{9}^{\Phi \Psi}=\llbracket \exists p \forall a b[\Phi \wedge \Psi \rightarrow B(p a b)] \rightarrow \exists p \forall a b[\Phi \wedge \Psi \rightarrow B(a p b)] \rrbracket$ where $\Phi, \Psi$ are members of $\mathbf{L}(\boldsymbol{B})$ such that $\boldsymbol{p}, \boldsymbol{b}$ do not occur free in $\Phi$, and $\boldsymbol{p}, \boldsymbol{a}$ do not occur free in $\Psi$. Elementary continuity axiom schema.

We begin with the representation problem, which we interpret here as the problem of providing a simple algebraic characterization of the class of all models of $\mathbf{G A}_{2}$. We first construct a special class of possible models for $\mathbf{G A}_{\mathbf{2}}$ by means of the following procedure. Let $\widetilde{\mho}=\langle F,+, \cdot, \leqslant\rangle$ be an ordered field; the elements 0 and 1 of $F$ and the binary operation - on elements of $F$ are defined in the usual way. Consider the two-dimensional linear space over $\mathfrak{F}$, i.e., the set $F \times F$ with two operations:

$$
\begin{gathered}
\langle x, y\rangle \oplus\left\langle x^{\prime}, y^{\prime}\right\rangle=\left\langle x+x^{\prime}, y+y^{\prime}\right\rangle, \\
\langle x, y\rangle \odot z=\langle x \cdot z, y \cdot z\rangle .
\end{gathered}
$$

DEFINITION 1.5. By the two-dimensional affine Cartesian space, or simply the affine plane, over $\mathfrak{F}$ we mean the relational system $\mathfrak{N}_{2}(\mathfrak{F})=\left\langle A_{\mathfrak{F}}, B_{\mathfrak{F}}\right\rangle$ where $A_{\mathfrak{F}}=F \times F$ and $B_{\overparen{F}}$ is a ternary relation on $A_{\overparen{F}}$ defined by the following stipulation: for any points $a, b, c \in A_{\tilde{F}}$,

$B_{\overparen{F}}(a b c)$ if, for some $x \in F, 0 \leqslant x \leqslant 1$ and $b=[a \odot(1-x)] \oplus(c \odot x)$.

In referring to $\mathfrak{R}_{2}(\mathfrak{\&})$ we shall use ordinary geometrical terminology. In particular we shall often refer to the set $A_{\mathfrak{F}}$ as a plane and to its elements as points. We assume that it is known which subsets of $A_{\mathfrak{F}}$ are called lines, halflines, interiors of triangles, polygons, etc. We obtain a natural topology on $A_{\mathfrak{F}}$ by taking the family of all interiors of triangles as the base for open sets. A set $S \subseteq A_{\mathbb{F}}$ is said to be convex if, for any $a, b, c$ with $a, c \in S$ and $B_{\widetilde{\mho}}(a b c)$ we have $b \in S$. We shall also need the notion of a weakly convex set: a set $S$ is weakly convex if, for any four distinct points $a, b, c, d \in S$, whenever the point $p$, in the intersection of the two distinct lines $K$ and $L$ passing, respectively, through $a, b$ and $c, d$, satisfies the condition $B_{\mathbb{F}}(a p b)$, then $p \in S$. The set $S$ is called non-linear if it contains three points $a, b, c$ which are non-collinear. In this paper, when referring to a convex set, we shall mean always a set which is convex, open and non-linear.

Now let $S$ be any subset of $A_{\mathscr{r}}$. The relational structure formed by the set $S$ and the relation $B_{\mathbb{F}}$ restricted to the points of $S$ (i. e. the intersection of $B_{\mathfrak{F}}$ and $S \times S \times S$ ), will be denoted by $\mathfrak{R}_{2}(\mathfrak{F}, S)$ and will be called the $S$-restricted affine plane over $\mathfrak{F}$. When applying these constructions, we often take for $\mathfrak{F}$ the ordered field $\mathfrak{R}$ of real numbers.

We shall prove a representation theorem to the following effect: for any model $\mathfrak{A}=\langle A, B\rangle$ of $\mathbf{G A}_{2}$ there is a real-closed field $\mathfrak{F}$ and a set $S$ convex in $\mathfrak{N}_{2}(\mathfrak{F})$ such that $\mathfrak{Q} \mathfrak{C}$ is isomorphic to $\mathfrak{Q}_{2}(\mathfrak{F}, S)$.

$\S 2$. Construction of an ordered real-closed field. The first step in the proof of the representation theorem is the construction of a field in an arbitrary model of weak general affine geometry (see Szczerba [11]). This construction is analogous to the well-known construction of a field in models of Euclidean metric geometry, or affine (descriptive) geometry (see e.g. Veblen [18]).

Let $\mathfrak{A}=(A, B)$ be an arbitrary, but fixed, model of $\mathbf{G A}_{2}$. Assume further that $e_{0}, e_{1}, e_{\omega}, e_{\infty}$ are arbitrary, but again fixed, points of $A$ satisfying the condition

DEFINITIUN 2.2. The line

$$
\neg L\left(e_{0} e_{1} e_{\omega}\right) \wedge B\left(e_{0} e_{1} e_{\infty}\right) \wedge \neg e_{1}=e_{\infty} .
$$

$$
L_{\infty}=\left\{a: L\left(a e_{\infty} e_{\omega}\right)\right\}
$$

will be called the line at pseudo-infinity.

As is well known, in what could be called the "projective approach" to Euclidean geometry, two lines in this geometry are parallel iff they intersect the line at infinity at the same point. By analogy we can introduce the notion of pseudo-parallelism:

Definttion 2.3. $a b \|_{e_{\infty} e_{\omega}} c d$ if there is a $p$ such that $p \in L_{\infty}$ and $L(a b p)$ and $L(c d p)$ (see Fig. 2.4).

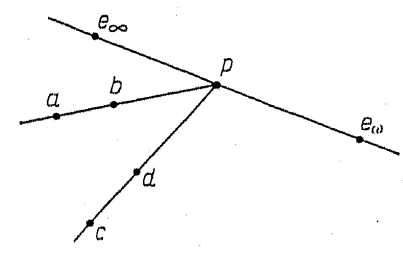

Fig. 2.4

The parameters $e_{\infty}, e_{\omega}$, being fixed, will usually be omitted. Thus we shall write simply $a b \| c d$ instead of $a b \|_{e_{\infty} e_{\omega}} c d$. We shall omit parameters in subsequent definitions. 
In Euclidean geometry the notion of congruence of vectors lying on a given line may be defined by means of parallelism (see Fig. 2.5a). Here we introduce the notion of pseudo-congruence of vectors (see Fig. 2.5b) by means of pseudoparallelism: a)

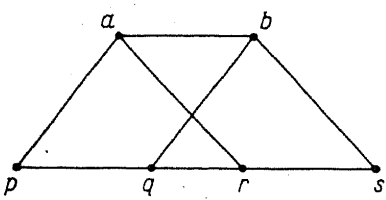

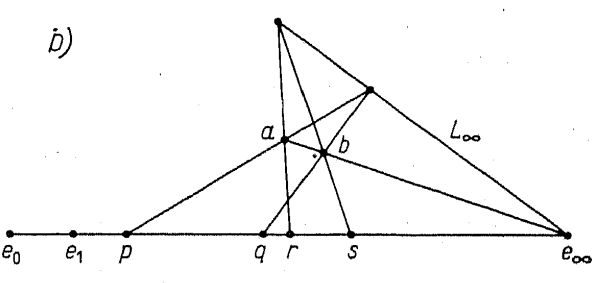

Fig. 2.5
Definition 2.6. $p q \equiv r s$ if there are $a, b$ such that $p, q, r, s \in\left[e_{0} \dot{e}_{\infty}\right)$, $a, b \notin L_{\infty}, a \notin L\left(e_{0} e_{\infty}\right) ; a p\|b q, a r\| b s$ and $e_{0} e_{1} \| a b$.

Similarly, in analogy to the Euclidean relation of proportionality (see Fig. 2.7a) where the pair of segments. $\left\langle e_{0}, p\right\rangle$ and $\left\langle e_{0}, q\right\rangle$ is proportional to the pair $\left\langle e_{0}, r\right\rangle$ and $\left\langle e_{0}, s\right\rangle$, we may introduce the notion of pseudo-proportionality (see Fig. 2.7b): a)

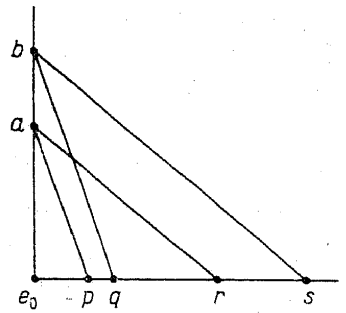

b)

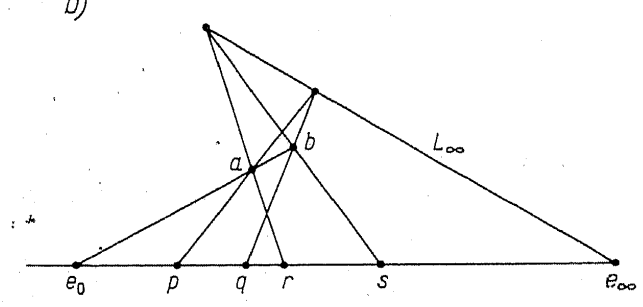

Fig. 2.7
Definition 2.8. $P(p, q, r, s)$ iff there are $a, b$ such that $p, q, r, s \in\left[e_{0} e_{\alpha}\right)$, $a \notin L\left(e_{0} e_{\infty}\right), a p\|b q, a r\| b s$ and $L\left(e_{0} a b\right)$.

By means of pseudo-congruence and pseudo-proportionality we define two. binary operations, + and $\cdot$, and a binary relation $\leqslant$ on the segment $\left[e_{0} e_{\infty}\right)$ : Definition 2.9. For any points $p, q \in\left[e_{0} e_{\infty}\right]$

(i) $p+q$ is the unique point $r$ such that $e_{0} p \equiv q r$,

(ii) $p \cdot q$ is the unique point $r$ such that $P\left(e_{1} p q r\right)$,

(iii) $p \leqslant q$ iff $B\left(e_{0} p q\right)$.

We let

$$
\mathfrak{H}_{A}=\langle H, 0,1,+, \cdot, \leqslant\rangle
$$

(where $H=\left[e_{0} e_{\infty}\right), 0=e_{0}, 1=e_{1}$ ).
In Szczerba [9] and [11] various properties of $\mathfrak{S}_{\mathfrak{X}}$ have been established, such as commutativity and associativity for + , associativity of ', distributivity of - over + It is proved that $p \leqslant q$ if and only if $p+x=q$ for some $x$. To get an algebra in which the equation $p+x=q$ is always solvable, we take the algebra $\mathfrak{G}_{\mathfrak{g}}$ of differences over $\mathfrak{J}_{\mathfrak{Q}}$.

DEFINITION 2.10. We set

where

$$
\left(\mathfrak{S}_{\mathfrak{Q}}=\langle H \times H,\langle 0,0\rangle,\langle 1,0\rangle, \oplus, \odot, \leqslant\rangle\right.
$$

$$
\begin{aligned}
& \langle p, q\rangle \oplus\langle r, s\rangle=\langle p+r, q+s\rangle . \\
& \langle p, q\rangle \odot\langle r, s\rangle=\langle p \cdot r+q \cdot s, p \cdot s+q \cdot r\rangle, \\
& \langle p, q\rangle \leqslant\langle r, s\rangle \text { if } \quad p+s \leqslant q+r:
\end{aligned}
$$

We also agree to write

$$
\langle p, q\rangle \approx\langle r, s\rangle \text { iff } p+s=q+r .
$$

From 2.10 it follows that $\approx$ is a congruence relation on the algebraic structure $\mathfrak{b}_{\mathfrak{Q}}$. Hence, as is well known from the general theory of algebraic structures (see HenkinMonk-Tarski [1] pp. $73 \mathrm{ff}$.), we can construct the corresponding quotient structure, which we shall denote by $\mathfrak{F}_{\mathfrak{Q}}$.

DEFINITION 2.11. $\mathfrak{F}_{\mathfrak{Q I}}=\left\langle F_{\mathfrak{Q}}, 0,1,+, \cdot, \leqslant\right\rangle=\mathfrak{G}_{\mathfrak{Q}} / \approx$.

The use of the same symbols for relations and operations in $\mathfrak{J}_{\mathfrak{Q}}$ and $\mathfrak{F}_{\mathfrak{I}}$ will not lead to any misunderstanding.

It has to be pointed out that the algebras $\mathfrak{S}_{\mathfrak{Q}}$ and $\mathfrak{F}_{\mathfrak{Q}}$ both depend on the parameters $e_{0}, e_{1}, e_{\infty}, e_{\omega}$. It has been proved, however, (see Szczerba [11]) that this dependence is inessential: corresponding algebras defined for different sets of parameters satisfying condition (2.1) are isomorphic. For this reason the fact that the dependence is not explicitly shown in our symbolism will not create any confusion.

THEOREM 2.12. If $\mathfrak{Q}$ is a model of $\mathbf{G A}_{2}$ then

(i) $\tilde{F}_{\mathrm{q}}$ is a field,

(ii) $\{x: 0 \leqslant x\}$ is a subuniverse of $\mathfrak{F}_{\mathscr{P}}$,

(iii) $\mathfrak{S}_{\mathbb{N}}$ is isomorphic to the subalgebra of $\widetilde{F}_{\mathfrak{I}}$ induced by $\{x: 0 \leqslant x\}$, i.e., to $\tilde{F}_{21} \mid\{x: 0 \leqslant x\}$.

The proof, may be found in Szczerba [11].

Our next task is to prove that, under the assumption of Theorem $2.12, \mathfrak{F}_{\mathfrak{g}}$ is a real-closed field (see Theorem 2.19). In proving this, we shall use a method permitting us to express various facts about $\mathfrak{J}_{\mathfrak{Q}}$ and $\mathfrak{F}_{\mathfrak{Q}}$ in the language of $\mathfrak{Q}$. The method is provided by a sequence of Lemmas, 2.14 through 2.18 , which establish connections between formulas in the languages of $\mathfrak{A}, \mathfrak{L}_{\mathfrak{Q}}$ and $\mathfrak{F}_{\mathfrak{Q}}$.

In dealing with the set of formulas $\mathbf{L}(\mathbf{0}, \mathbf{1},+, \cdot, \leqslant)$ (the common language of $\mathfrak{S}_{\mathscr{V}}$ and $\mathfrak{F}_{\mathfrak{y}}$ ) we find it convenient to single out the set $\mathbf{L}^{\prime}$ of those formulas in which all the atomic subformulas are of the form

$$
v_{i}=0, v_{i}=1, v_{i}=v_{j}, v_{i}+v_{j}=v_{k}, v_{i} \cdot v_{j}=v_{k}, v_{i} \leqslant v_{k} .
$$


Thus, for example, the formula $\boldsymbol{x}+\boldsymbol{y}=\boldsymbol{y}+\boldsymbol{x}$ does not belong to $\mathrm{L}^{\prime}$. As is well known (see e. g. Robinson [6], Section 9.1) there is a well-determined, recursive unary operation $\nabla$ which correlates with every formula $\Phi \in \mathbf{L}(\mathbf{0}, \mathbf{1},+, \cdot, \leqslant)$ the formula $\Phi^{\nabla} \in \mathbf{L}^{\prime}$ in such a way that $\Phi$ and $\Phi^{\nabla}$ are logically equivalent and both formulas have the same variables. The operator $\nabla$ and set $\mathbf{L}^{\prime}$ will be used in formulating the next two definitions, 2.13 and 2.16

Definition 2.13. Let $\boldsymbol{H}$ be the formula

$$
H=[B(a x c) \wedge \neg x=c]
$$

and let $\mathbf{H}$ be the unique function mapping $\mathbf{L}(\mathbf{0}, \mathbf{1},+, \cdot, \leqslant)$ into $\mathbf{L}(\boldsymbol{B})$ which satisfies the following conditions:

(i) $\mathbf{H}\left(\boldsymbol{v}_{i}=\mathbf{0}\right)=\left[\boldsymbol{v}_{i+8}=a\right]$

(ii) $\mathbf{H}\left(\boldsymbol{v}_{i}=\mathbf{1}\right)=\left[\boldsymbol{v}_{i+8}=\boldsymbol{b}\right]$

(iii) $\mathbf{H}\left(v_{i}=v_{j}\right)=\left[v_{i+8}=v_{j+8}\right]$

(iv) $\mathrm{H}\left(v_{i} \leqslant v_{j}\right)=\left[H\left(v_{i+8}\right) \wedge H\left(v_{j+8}\right) \wedge B\left(a v_{i+8} v_{j+8}\right)\right]$

(v) $\boldsymbol{H}\left(\boldsymbol{v}_{i}+\boldsymbol{v}_{j}=\boldsymbol{v}_{k}\right)=\left[\boldsymbol{H}\left(\boldsymbol{v}_{i+8}\right) \wedge \boldsymbol{H}\left(\boldsymbol{v}_{j+8}\right) \wedge \boldsymbol{H}\left(\boldsymbol{v}_{k+8}\right) \wedge\right.$ $\wedge \exists v_{4} v_{5} v_{6} v_{7}\left[\neg B\left(c v_{4} d\right) \wedge \neg B\left(c v_{5} d\right) \wedge B\left(c v_{6} d\right) \wedge B\left(c v_{7} d\right) \wedge\right.$ $\left.\left.\wedge B\left(a v_{5} v_{6}\right) \wedge B\left(c v_{4} v_{5}\right) \wedge B\left(v_{i+8} v_{4} v_{6}\right) \wedge B\left(v_{j+8} v_{5} v_{7}\right) \wedge B\left(v_{k+8} v_{4} v_{7}\right)\right]\right]$,

(vi) $H\left(v_{i} \cdot v_{j}=v_{k}\right)=\left[H\left(v_{i+8}\right) \wedge H\left(v_{j+8}\right) \wedge H\left(v_{k+8}\right) \wedge \exists v_{4} v_{5} v_{6} v_{7}\left[\neg B\left(c v_{4} d\right) \wedge\right.\right.$ $\wedge \neg B\left(c v_{5} d\right) \wedge B\left(c v_{6} d\right) \wedge B\left(c v_{7} d\right) \wedge B\left(a v_{4} v_{5}\right) \wedge B\left(b v_{4} v_{6}\right) \wedge B\left(v_{i+8} v_{4} v_{7}\right) \wedge$ $\left.\left.\wedge B\left(v_{j+8} v_{5} v_{6}\right) \wedge B\left(v_{k+8} v_{5} v_{7}\right)\right]\right]$

(vii) $\mathbf{H}(\neg \Phi)=\neg \mathbf{H}(\Phi)$ for any $\Phi \in \mathbf{L}^{\prime}$.

(viii) $\mathbf{H}(\Phi \vee \Phi)=[\mathbf{H}(\Phi) \vee \mathbf{H}(\Psi)]$ for any $\Phi, \Psi \in \mathbf{L}^{\prime}$,

(ix) $\mathbf{H}\left(\exists v_{i} \Phi\right)=\left[\exists v_{i+8} H\left(v_{i+8}\right) \wedge H(\Phi)\right]$ for any $\Phi \in \mathbf{L}^{\prime}$

(x) $\mathbf{H}(\Phi)=\mathbf{H}\left(\Phi^{\nabla}\right)$ for any $\Phi \in \mathbf{L}(\mathbf{0}, \mathbf{1},+, \cdot, \leqslant) \backslash \mathbf{L}^{\prime}$ (cf. Definition 2.9).

In Lemmas $2.14,2.17$ and 2.18 we shall assume that $\Phi \in \mathbf{L}(\mathbf{0}, \mathbf{1},+, \cdot, \leqslant)$ and $v_{i_{0}}, \ldots, v_{i_{n}}$ is a sequence of variables without repetitions containing all free variables of $\Phi$.

LemmA 2.14. Let $v_{0}, \ldots, v_{n}$ be a sequence of elements of $\mathfrak{S}_{\mathfrak{q}}$. Then

$$
\mathfrak{S}_{\mathfrak{Q}} \vDash \Phi\left[\begin{array}{lll}
\boldsymbol{v}_{i_{0}} & \cdots & \boldsymbol{v}_{i_{n}} \\
v_{0} & \cdots & v_{n}
\end{array}\right] \quad \text { iff } \quad \mathfrak{Q} \vDash \mathbf{H}(\Phi)\left[\begin{array}{lllllll}
\boldsymbol{a} & \boldsymbol{b} & \boldsymbol{c} & \boldsymbol{d} & \boldsymbol{v}_{i_{0}+8} & \cdots & \boldsymbol{v}_{i_{n}+8} \\
e_{0} & e_{1} & e_{\infty} & e_{\omega} & v_{0} & \cdots & v_{n}
\end{array}\right] \text {. }
$$

Proof. By an easy induction on formulas in $\mathbf{L}(\mathbf{0}, \mathbf{1},+, \cdot, \leqslant)$ according to Definition 2.13.

From 2.14 we immediately get

Corollary 2.15. For any sentence $\Phi \in \mathbf{L}(\mathbf{0}, \mathbf{1},+, \cdot, \leqslant)$ we have

$$
\mathfrak{S}_{\mathfrak{I I}} \vDash \Phi \quad \text { iff } \quad \mathfrak{T} \vDash \mathbf{H}(\Phi)\left[\begin{array}{llll}
\boldsymbol{a} & \boldsymbol{b} & \boldsymbol{c} & \boldsymbol{d} \\
e_{0} & e_{1} & e_{\infty} & e_{\omega}
\end{array}\right] \text {. }
$$

Definition 2.16. Let $\mathbf{F}$ be the unique function mapping $\mathbf{L}(\mathbf{0}, \mathbf{1},+, \cdot, \leqslant)$ into itself and satisfying the following conditions: (i)-(x): (i) $\mathrm{F}\left(\boldsymbol{v}_{i}=\mathbf{0}\right)=\left[\boldsymbol{v}_{2 i}=v_{2 i+1}\right]$,

(ii) $\mathbf{F}\left(v_{l}=1\right)=\left[v_{2 i}=v_{2 i+1}+1\right]$,

(iii) $\mathbf{F}\left(v_{i}=v_{j}\right)=\left[v_{2 i}+v_{2 j+1}=v_{2 i+1}+v_{2 j}\right]$

(iv) $\mathrm{F}\left(v_{i} \leqslant v_{j}\right)=\left[v_{2 i}+v_{2 j+1} \leqslant v_{2 i+1}+v_{2 j}\right]$,

(v) $F\left(v_{l}+v_{j}=v_{k}\right)=\left[v_{2 i}+v_{2 j}+v_{2 k+1}=v_{2 i+1}+v_{2 j+1}+v_{2 k}\right]$,

(vi) $\mathbf{F}\left(\boldsymbol{v}_{i} \cdot v_{j}=v_{k}\right)=\left[v_{2 i} \cdot v_{2 j}+v_{2 i+1} \cdot v_{2 j+1}+v_{2 k+1}=v_{2 i+1} \cdot v_{2 j}+\right.$ $\left.+v_{2 i} \cdot v_{2 j+1}+v_{2 k}\right]$

(vii) $\mathbf{F}(\neg \Phi)=\neg \mathbf{F}(\Phi)$ for any $\Phi \in \mathbf{L}^{\prime}$,

(viii) $\mathbf{F}(\Phi \vee \Psi)=[\mathbf{F}(\Phi) \vee \mathbf{F}(\Psi)]$ for any $\Phi, \Psi \in \mathbf{L}^{\prime}$,

(ix) $\mathbf{F}\left(\exists v_{i} \Phi\right)=\left[\exists v_{2 l} \exists v_{2 i+1} \mathbf{F}(\Phi)\right]$ for any $\Phi \in \mathbf{L}^{\prime}$,

(x) $\mathbf{F}(\Phi)=\mathbf{F}\left(\Phi^{\nabla}\right)$ for any $\Phi \in \mathbf{L}(\mathbf{0}, \mathbf{1},+, \cdot, \leqslant) \backslash \mathbf{L}^{\prime}$.

Just as in case of Lemma 2.14 we prove by induction:

LEMMA 2.17. If $\left\langle\left.\left\langle x_{0}, y_{0}\right\rangle\right|_{\approx}, \ldots,\left.\left\langle x_{n}, y_{n}\right\rangle\right|_{\approx}\right\rangle$ is a sequence of elements of $\mathfrak{F}_{\mathfrak{W}}$, then

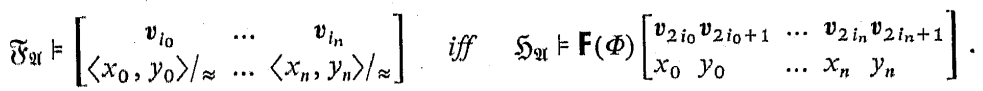

Lemmas 2.14 and 2.17 imply then

LEMMA 2.18. If $\left\langle\left\langle x_{0}, y_{0}\right\rangle / \approx, \ldots,\left\langle x_{n}, y_{n}\right\rangle / \approx\right\rangle$ is a sequence of elements of $\mathfrak{F}_{\mathfrak{M}}$,

$$
\mathfrak{F}_{\mathfrak{Q R}} \vDash \Phi\left[\begin{array}{ccc}
\boldsymbol{v}_{i_{0}} & \cdots & \boldsymbol{v}_{i_{n}} \\
\left\langle x_{0}, y_{0}\right\rangle / \approx & \cdots & \left\langle x_{n}, y_{n}\right\rangle / \approx
\end{array}\right]
$$

iff

$$
\mathfrak{Q} \vDash \operatorname{HF}(\Phi)\left[\begin{array}{lllllllll}
\boldsymbol{a} & \boldsymbol{b} & \boldsymbol{c} & \boldsymbol{d} & \boldsymbol{v}_{2 i_{0}+8} & \boldsymbol{v}_{2 i_{0}+9} & \ldots & \boldsymbol{v}_{2 i_{n}+8} & \boldsymbol{v}_{2 i_{n}+9} \\
e_{0} & e_{1} & e_{\infty} & e_{\omega} & x_{0} & y_{0} & \ldots & x_{n} & y_{n}
\end{array}\right] \text {. }
$$

Theorem 2.19. If $\mathfrak{Q}$ is a model of $\mathbf{G A}_{2}$, then $\mathfrak{F}_{\mathfrak{Q}}$ is a real-closed field.

Proof. We first prove that $\mathfrak{F}_{\mathscr{E}}$ is a model of all the sentences of the form

$$
F^{\Phi \Psi}=\llbracket \forall v_{0} \forall v_{2}\left(\Phi \wedge \Psi \rightarrow v_{0} \leqslant v_{2}\right) \rightarrow \exists v_{1} \forall v_{0} \forall v_{2}\left(\Phi \wedge \Psi \rightarrow v_{0} \leqslant v_{1} \leqslant v_{2}\right) \rrbracket
$$

where $\Phi$ and $\psi$ are any formulas in $\mathbf{L}(\mathbf{0}, \mathbf{1},+, \cdot, \leqslant)$ such that $\boldsymbol{v}_{0}$ and $\boldsymbol{v}_{1}$ do not occur free in $\Phi$, while $v_{1}$ and $v_{2}$ do not occur free in $\Psi$. The formula $\mathbf{H}\left(\boldsymbol{F}^{\Phi \Psi}\right)$ is logically equivalent to

$$
\begin{gathered}
\left(F^{\left(\Phi \Psi^{\prime}\right.}\right)^{\prime}=\left[\forall v_{8} \forall v_{10}\left[\boldsymbol{H}\left(\begin{array}{l}
v_{4} \\
v_{8}
\end{array}\right) \wedge H\left(\begin{array}{c}
v_{4} \\
v_{10}
\end{array}\right) \wedge H(\Phi) \wedge H(\Psi) \rightarrow B\left(v_{0} v_{8} v_{10}\right)\right]\right. \\
\left.\rightarrow \exists v_{9} \forall v_{8} \forall v_{10}\left[H\left(\begin{array}{l}
v_{4} \\
v_{8}
\end{array}\right) \wedge H\left(\begin{array}{l}
v_{4} \\
v_{9}
\end{array}\right) \wedge H(\Phi) \wedge H(\Psi) \rightarrow B\left(v_{0} v_{8} v_{9}\right) \wedge B\left(v_{0} v_{9} v_{10}\right) \wedge H\left(\begin{array}{l}
v_{4} \\
v_{9}
\end{array}\right)\right]\right] .
\end{gathered}
$$

Since it is easy to show by means of $\mathbf{A}_{9}^{\left.H^{(}()\right)} \mathbf{H}^{(\Psi)}$ that

$$
\mathfrak{Y} \vDash\left(F^{\Phi \Psi}\right)^{\prime}\left[\begin{array}{llll}
a & b & c & d \\
e_{0} & e_{1} & e_{\infty} & e_{\omega}
\end{array}\right],
$$


it follows by 2.15 that $\boldsymbol{F}^{\Phi \Psi}$ is true in $\mathfrak{h}_{\mathfrak{Y}}$. Since $\mathfrak{F}_{\mathfrak{T}}$ is isomorphic to $\widetilde{F}_{\mathfrak{r}} \mid\{x: 0 \leqslant x\}$ (see 2.12(ii)), it is easy to see that $F^{\Phi \Psi}$ is true in $\widetilde{F}_{2}$. We now prove that $\widetilde{F}_{21}$ is commutative. Consider two sets:

$X=\left\{x: x \leqslant 0\right.$ or, for every $u, v \in F_{\mathfrak{N}}$, if $0 \leqslant u \leqslant x$ and $0 \leqslant v \leqslant x$, then $\left.u \cdot v=v \cdot u\right\}$, $Z=\left\{z\right.$ : there are $\dot{u}, v \in F_{\mathfrak{Q}}$ such that $0 \leqslant u \leqslant z, 0 \leqslant v \leqslant z$, and $\left.u \cdot v \neq v \cdot u\right\}$.

We easily see that $x \leqslant z$ whenever $x \in X$ and $z \in Z$. Obviously the sets $X$ and $Z$ are both definable; hence we conclude that there is a $y$ such that

(1) $x \leqslant y$ and $y \leqslant z$ whenever $x \in X$ and $z \in Z$.

Now since $X \cup Z=F_{21}$ and $X$ is non-empty, we get $y-1 \in X$. Clearly $y+1$ $=(y-1)+2 \in X$. Hence there is a $y^{\prime} \in X$ such that $y<y^{\prime}$. From (1) it now follows that the set $Z$ is empty. Hence $\widetilde{F}_{\mathscr{T}}$ is a commutative field. Since in addition $\widetilde{F}_{\mathfrak{T}}$ is a model of all the elementary continuity axioms, this completes the proof (see Tarski [15]).

LEMMA 2.20. For every model $\mathfrak{Q}$ of $\mathbf{G A}_{2}$ there is an ordered, real-closed field $₹$ and a set $S$, nonlinear and weakly convex in $\mathfrak{Q R}_{2}(\mathfrak{F})$ and such that $\mathfrak{A}$ is isomorphic to $\mathfrak{H}_{2}(\mathfrak{F}, S)$.

A proof is given in detail in Szczerba [9] (cf. Theorem 8, Szczerba [11]). Here we shall give a brief outline of the proof.

Let $\mathfrak{I}=\langle A, B\rangle$ be a model of $\mathbf{G A}_{2}$. Consider the following relational structure

$$
\left\langle F_{\mathfrak{Y I}} \times F_{\mathfrak{Q I}} \times F_{\mathfrak{Q I}} \backslash\{\langle 0,0,0\rangle\}, L_{\mathfrak{P}}\right\rangle
$$

where

$$
L_{\mathfrak{P}}\left(\left\langle a_{0}, a_{1}, a_{2}\right\rangle,\left\langle b_{0}, b_{1}, b_{2}\right\rangle,\left\langle c_{0}, c_{1}, c_{2}\right\rangle\right) \quad \text { iff }\left|\begin{array}{lll}
a_{0} & a_{1} & a_{2} \\
b_{0} & b_{1} & b_{2} \\
c_{0} & c_{1} & c_{2}
\end{array}\right|=0 .
$$

Let $\left\langle a_{0}, a_{1}, a_{2}\right\rangle \sim\left\langle b_{0}, b_{1}, b_{2}\right\rangle$ iff there is an $x \neq 0$ such that $a_{i}=x \cdot b_{i}(i=0,1,2)$. The relation $\sim$ is a congruence relation on this relational structure. By the projective plane over the field $\mathfrak{F}_{91}$ we mean

$$
\mathfrak{P}\left(\widetilde{\mho}_{\mathfrak{I P}}\right)=\left\langle F_{\mathfrak{P l}} \times F_{\mathfrak{P I}} \times F_{\mathscr{P I}} \backslash\{\langle 0,0,0\rangle\}, L_{\Re}\right\rangle / \sim .
$$

We define a mapping $f$ of $A$ into the projective plane which will help us in defining Cartesian coordinates. We first put $f\left(e_{0}\right)=\left.\langle 1,0,0\rangle\right|_{\sim}, f\left(e_{\infty}\right)=\left.\langle 0,1,0\rangle\right|_{\sim}$ and $f\left(e_{\omega}\right)=\langle 0,0,1\rangle / \sim$. Let $e^{\prime}$ be any point with $B\left(e^{\prime} e_{\omega} e_{\infty}\right)$ and $e^{\prime} \neq e_{\omega}$, we put $f\left(e^{\prime}\right)=\langle 0,1,-1\rangle / \sim$. If $e^{\prime \prime} \in\left(e_{0} e_{\omega}\right) \cap\left(e_{1} e^{\prime}\right)$ and $e^{\prime \prime \prime} \in\left(e_{1} e_{\omega}\right) \cap\left(e^{\prime \prime} e_{\omega s}\right)$, we put $f\left(e^{\prime \prime}\right)=\langle 1,0,1\rangle / \sim$ and $f\left(e^{\prime \prime \prime}\right)=\langle 1,1,1\rangle / \sim$ (see Fig. 2.21). Next we define $f$ for the points in the segment $\left(e_{0} e_{\infty}\right)$ :

$$
f(p)=\left\langle 1,\langle p, 0\rangle /_{\approx}, 0\right\rangle / \sim .
$$

Recall that the elements of the field $\widetilde{\mho}_{\mathfrak{T}}$ are equivalence classes of pairs of points.

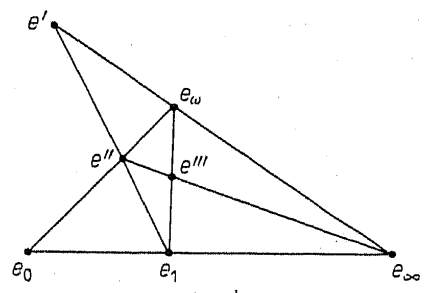

Fig. 2.21

The mapping $f$ is defined on the segment $\left(e_{0} e_{\omega}\right)$ by means of a projection with the centre at a point $e^{\prime}:$ for $p \in\left(e_{0} e_{\omega}\right)$ we put

$$
f(p)=\left\langle 1,0,\left\langle p^{\prime}, 0\right\rangle / \approx\right\rangle / \sim
$$

where $p^{\prime} \in\left(e_{0} e_{\infty}\right) \cap L\left(e^{\prime} p\right)$. Similarly, by a projection with the centre $e^{\prime \prime}$, we define the function $f$ for points in $\left(e_{\infty} e_{\omega t}\right)$.

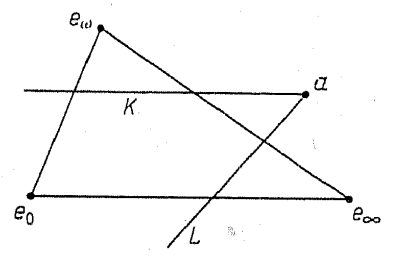

Fig. 2.22

To define the mapping $f$ for an arbitrary point $p$ we make the following construction. Let $K$ and $L$ be two distinct lines passing through the point $a$ such that each of them intersects two of the segments $\left(e_{0} e_{\infty}\right),\left(e_{0} e_{\omega}\right)$ and $\left(e_{\infty} e_{\omega}\right)$. Let $q^{K}, r^{K}$ and $q^{L}, r^{L}$ be the points of intersection for $K$ and $L$, respectively (see Fig. 2.22). Let $f\left(q^{K}\right)=\left\langle q_{0}^{K}, q_{1}^{K}, q_{2}^{K}\right\rangle / \sim, f\left(r^{K}\right)=\left\langle r_{0}^{K}, r_{1}^{K}, r_{2}^{K}\right\rangle / \sim, f\left(q^{L}\right)=\left\langle q_{0}^{L}, q_{1}^{L}, q_{2}^{L}\right\rangle / \sim$ and $f\left(r^{L}\right)=\left\langle r_{0}^{L}, r_{1}^{L}, r_{2}^{L}\right\rangle / \sim$. We put $f(a)=\left\langle a_{0}, a_{1}, a_{2}\right\rangle / \sim$ iff

$$
\left|\begin{array}{lll}
a_{0} & a_{1} & a_{2} \\
q_{0}^{K} & q_{1}^{K} & q_{2}^{K} \\
r_{0}^{K} & r_{1}^{K} & r_{2}^{K}
\end{array}\right|=\left|\begin{array}{ccc}
a_{0} & a_{1} & a_{2} \\
q_{0}^{L} & q_{1}^{L} & q_{2}^{L} \\
r_{0}^{L} & r_{1}^{L} & r_{2}^{L}
\end{array}\right|=0 .
$$

To obtain the Cartesian coordinates, we have to use a projective transformation (see Szczerba [9] and also [12]) mapping the line at pseudo-infinity into a line outside the set $f^{*} A$, the $f$-image of $A$. For some models of general affine geometry without the continuity schema this requires an extension of the field $\widetilde{F}_{\mathfrak{r}}$ by means of the method developed in Szczerba [10]. The extended field is elementarily equivalent to $\widetilde{F}_{2}$ and therefore is real-closed. In the next section we shall prove Lemma 3.1, which implies that in fact for models of general affine geometry with the continuity schema there is no need to pass to an extension of the field $\widetilde{F}_{\mathfrak{r}}$. 
Let us assume that $\Phi$ is a universal sentence true in $\mathfrak{X}_{2}(\mathfrak{R})$, the affine plane over the field of real numbers; here and in the subsequent discussion "'R" is used to denote the (ordered) field of real numbers. For any real-closed field $\mathfrak{F}$, $\mathfrak{F}$ is elementarily equivalent to $\mathfrak{R}$, and hence $\Phi$ is true in $\mathfrak{U}_{2}(\mathfrak{F})$.

Therefore, by Lemma 3.3, $\Phi$ is true in any model of $\mathbf{G A}_{2}$.

LEMMA 2.23. If a universal sentence $\Phi$ is true in $\mathfrak{R}_{2}(\mathfrak{R})$, then it is true in any model of $\mathbf{G A}_{2}$.

This lemma is a particular case of a general result stated below in Theorem 6.11.

Remark 2.24. In this section we have constructed a field and introduced the Cartesian coordinates. Implicitly, this gives us a relation of equidistance which depends essentially on the parameters $e_{0}, e_{1}, e_{\infty}, e_{\omega}$. For different sets of parameters we get, in general, different equidistance relations. This shows, however, that a (non-relativized) relation $D$ of equidistance is not definable in terms of $B$ alone. It is interesting to note that essentially the same argument appears in certain investigations in the foundations of geometry which apparently serve a directly opposite goal: namely, the establishment of metrical geometry as a part of affine geometry (see e.g. Veblen [18]).

§ 3. Representation problems. Our next task is to prove that it is possible to choose the field $\mathbb{F}$ mentioned in Lemma 2.20 in such a way that the set $S$ will be convex in $\mathfrak{I}_{2}(\widetilde{F})$.

To make some formulations easier, we introduce the following abbreviation:

$$
\boldsymbol{L}=\boldsymbol{B}(\boldsymbol{x y z}) \vee \boldsymbol{B}(\boldsymbol{y z x}) \vee \boldsymbol{B}(z x y) .
$$

LEMMA 3.1. If $\mathfrak{Q}$ is a model of $\mathbf{G A}_{2}$, then $\mathfrak{U}$ is a model of the sentence

\section{$S=\exists a b c d \forall p q r s t[L(a b p) \wedge L(b c q) \wedge L(c d r) \wedge L(d a s) \wedge L(a t c) \wedge L(p t r) \wedge$}

$$
\wedge L(s t q) \wedge L(p s v) \wedge L(q r v) \rightarrow p=s \vee q=r] .
$$

(see Fig. 3.2)

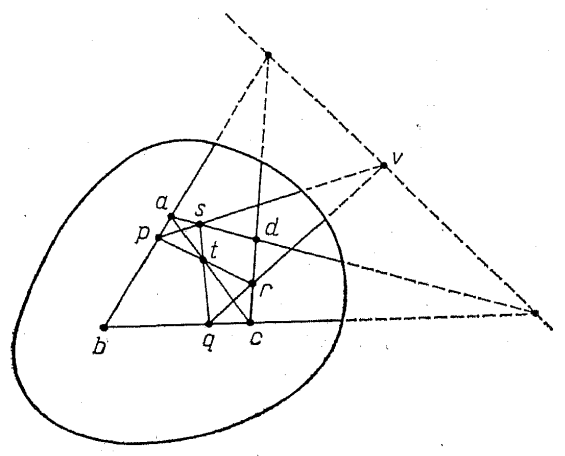

Fig. 3.2
Proof. Let $\mathfrak{Q}=\langle A, B\rangle$. By axiom $\mathbf{A}_{1}$, there are in $A$ three noncollinear points $a, b, c^{\prime}$. By $\mathbf{A}_{4}$ there is a point $a^{\prime}$ different from $b$ and such that $B\left(a^{\prime} b a\right)$, and a point $d^{\prime}$ different from $a$ and such that $B\left(d^{\prime} a c^{\prime}\right)$ (see Fig. 3.3). Thus, by axiom $\mathbf{A}_{5}$, we get a point $c$ such that $B\left(c^{\prime} b c\right)$ and $B\left(d^{\prime} c a^{\prime}\right)$.

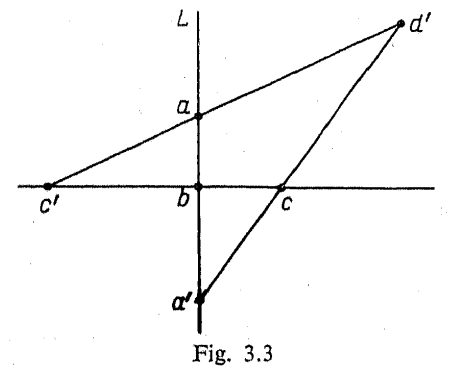

We now show that there is a line through the point $c$ disjoint with the line $L(a b)$. Indeed, we shall prove that there is a point $d^{\prime \prime}$ such that $B\left(c^{\prime} a d^{\prime \prime}\right)$ and. $L(a b) \cap L\left(c d^{\prime \prime}\right)=\varnothing$. Suppose that

$$
\begin{aligned}
& X=\left\{x: B\left(c^{\prime} a x\right) \text { and there is } w \text { such that } B(b a w) \text { and } L(c w x)\right\}, \\
& Y=\left\{y: B\left(c^{\prime} a y\right) \text { and there is } w \text { such that } B(a b w) \text { and } L(c w y)\right\} .
\end{aligned}
$$

It is easy to see that both sets are definable in $\mathbf{L}(\boldsymbol{B})$. Since $a \in X$ and $d^{\prime} \in Y$, neither set is empty, Moreover, by Lemma 2.23, if $x \in X$ and $y \in Y$ then $B(a x y)$. Therefore we may use a suitable instance of elementary continuity axiom $\mathbf{A}_{9}^{\mathbf{m}}$ to prove the existence of a $d^{\prime \prime}$ such that if $x \in X$ and $y \in Y$ then $B\left(x d^{\prime \prime} y\right)$. If $d^{\prime \prime} \in Y$ then by means. of Pasch's axiom $\mathbf{A}_{5}$ (see Fig. 3.4) there is an $y \in Y$ such that $B\left(\right.$ ayd $\left.d^{\prime \prime}\right)$ and $y \neq d^{\prime \prime}$. This is impossible, and therefore $d^{\prime \prime} \notin Y$. Similarly $d^{\prime \prime} \notin X$. By Lemma 2.23 it follows that the line $L\left(d^{\prime \prime} c\right)$ does not intersect the segment $(a b)$, and thus we have

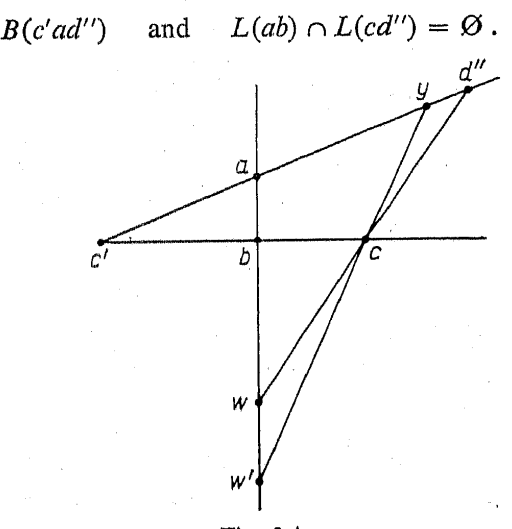


Let us consider the following property:

$$
Z\left(d^{\prime \prime \prime} p q r s t v\right) \quad \text { iff } \quad L(a b p), L(b c q), L\left(c d^{\prime \prime \prime} r\right), L\left(d^{\prime \prime \prime} a s\right), L(a t c)
$$

(see Fig. 3.5).

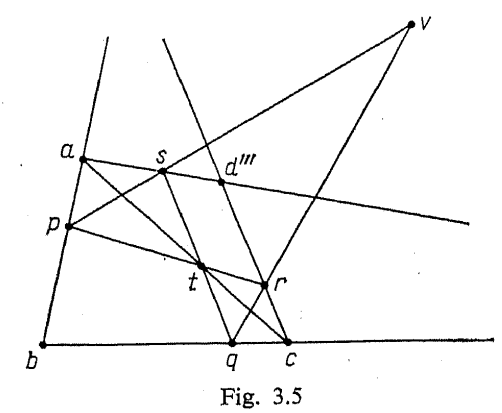

(2) If $Z\left(d^{\prime \prime \prime} p q r s t v\right)$, then there are $p^{\prime}, q^{\prime}, r^{\prime}, s^{\prime}, t^{\prime}$ such that $Z\left(d^{\prime \prime \prime} p^{\prime} q^{\prime} r^{\prime} s^{\prime} t^{\prime} v\right)$ and $B\left(a t^{\prime} c\right)$ and $B\left(b q^{\prime} c\right)$.

To prove this, a number of cases must be discussed. We shall prove (2) for the case:

$B(b c q)$ and $B(r q v)$ and $B(s q t)$

(see Fig. 3.6). The proofs of the remaining cases are analogous.

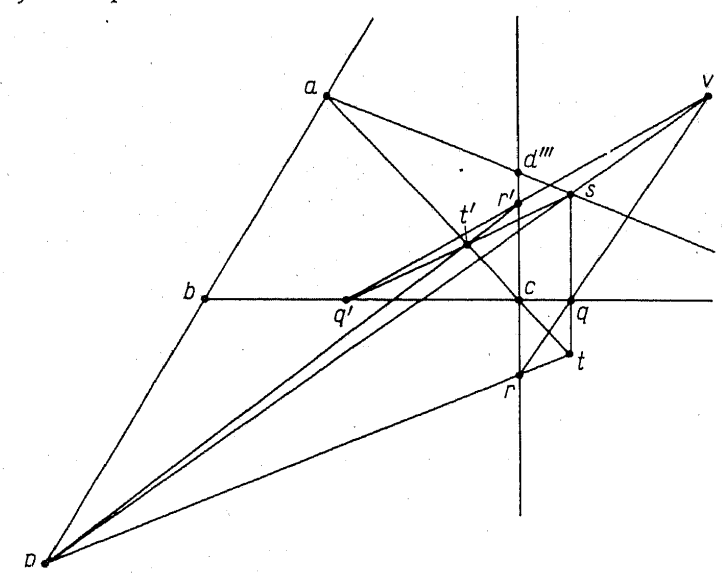

Fig. 3.6

Let $q^{\prime}$ be any point in the open segment $(b c)$. By Pasch's axiom, $\mathbf{A}_{5}$, we get a point $r^{\prime}$ such that $B\left(r c r^{\prime}\right)$ and $B\left(q^{\prime} r^{\prime} v\right)$. Similarly there is a point $t^{\prime}$ such that $B\left(s t^{\prime} q^{\prime}\right)$ and $B\left(t c t^{\prime}\right)$. Employing the analytical method one quickly establishes $L\left(p t^{\prime} r^{\prime}\right)$ and $B\left(a t^{\prime} c\right)$, from which (2) follows.
Let us now consider the sets (see Fig. 3.7)

$X=\left\{x: B\left(c x d^{\prime \prime}\right)\right.$ and there are $p, q, r, s, t, v, w$ such that $Z$ (xpqrstv), $L\left(c d^{\prime \prime} w\right)$ and $\left.B(b w v)\right\}$

$Y=\left\{y: B\left(c y d^{\prime \prime}\right)\right.$ and there are $p, q, r, s, t, v, w$ such that $Z($ ypqrstv $), L(a b w)$ and $B(c w v)\}$

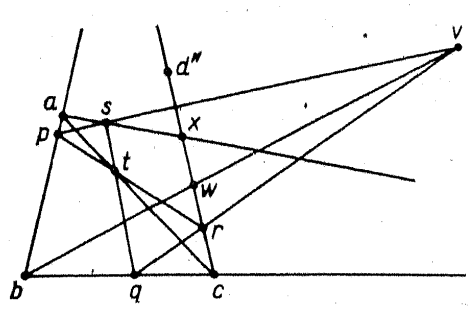

$x$

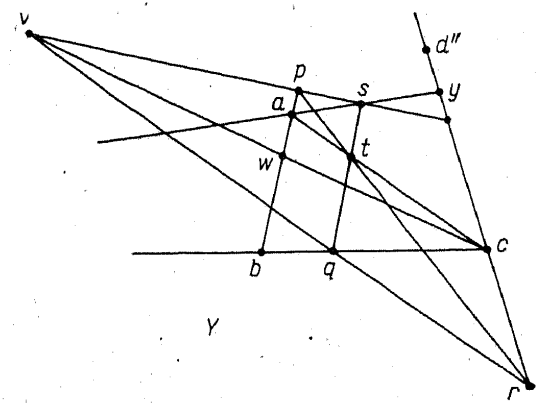

Fig. 3.7
If we order the segment $\left[c d^{\prime \prime}\right]$ in the natural way from the point $c$ to the point $d^{\prime \prime}$, then we can speak about a last element of the set $X$. In fact, $X$ does not contain a last element and $Y$ does not contain a first one.

(3) For any $x \in X$ there is an $x^{\prime} \in X$ such that $B\left(a x x^{\prime}\right)$ and $x \neq x^{\prime}$.

(4) For any $y \in Y$ there is a $y^{\prime} \in Y$ such that $B\left(c y^{\prime} y\right)$ and $y^{\prime} \neq y$.

We show (3). Since $x \in X$, we have $B\left(c x d^{\prime \prime}\right)$, and there are $p, q, r, s, t, r, v$ such that $Z$ (xpqrstv) and a point $w$ such that $L\left(c w d^{\prime \prime}\right)$ and $B(b w v)$. By (2) we may assume that $B(a t c)$ and $B(b q c)$ (see Fig. 3.8). This implies that $B(q r v)$. Let $v^{\prime}$ be any point such that $v \neq v^{\prime}$ and $B\left(b v v^{\prime}\right)$. From Pasch's axiom $\mathbf{A}_{\mathbf{s}}$ it follows that there is a point $r^{\prime}$ for which $B\left(w r r^{\prime}\right)$ and $B\left(q r^{\prime} v^{\prime}\right)$. Because $B\left(r r^{\prime} c\right)$, there is a point $p^{\prime}$ such that $B\left(a p^{\prime} p\right)$ and $B\left(p^{\prime} t r^{\prime}\right)$. Since $B\left(p^{\prime} t r^{\prime}\right)$ and $B\left(q r^{\prime} v^{\prime}\right)$, by $\mathbf{A}_{5}$, there is an $s^{\prime}$ such that $B\left(q t s^{\prime}\right)$ and $B\left(p^{\prime} s^{\prime} v^{\prime}\right)$. By the weak convexity of $A$ in $\mathfrak{R}_{2}\left(\mathfrak{F}_{\mathfrak{q}}\right)$ there is a point $x^{\prime} \neq x$ for which $B\left(c x x^{\prime}\right)$ and $x^{\prime} \in X$. We may prove (4) in a similar fashion.

By using Lemma 2.23, it is not difficult to show that $x \in X$ and $y \in Y$ implies $B(c x y)$. Thus by an appropriate instance of the elementary continuity axiom schema there is a point $d$ such that for any $x \in X$ and $y \in Y$ we have $B(x d y)$. Again by means of Lemma 2.23 the points $a, b, c, d$, must satisfy the following condition: $Z(d p q r s t v)$ does not hold for any $p, q, r, s, t, v$. Therefore $\mathfrak{A}$ is a model of a sentence $S$ and the proof is complete.

The intuitive meaning of sentence $S$ can be expressed as follows: there is a line in a projective plane over the field outside the universe of the model $\mathfrak{R}$. Hence there is no need to extend the field $\mathfrak{F}_{91}$ in the proof of Lemma 2.20 .

By Lemma 3.1 and Szczerba [11], Theorem 5 we obtain:

2- Fundamenta Mathemáticae CV/3 


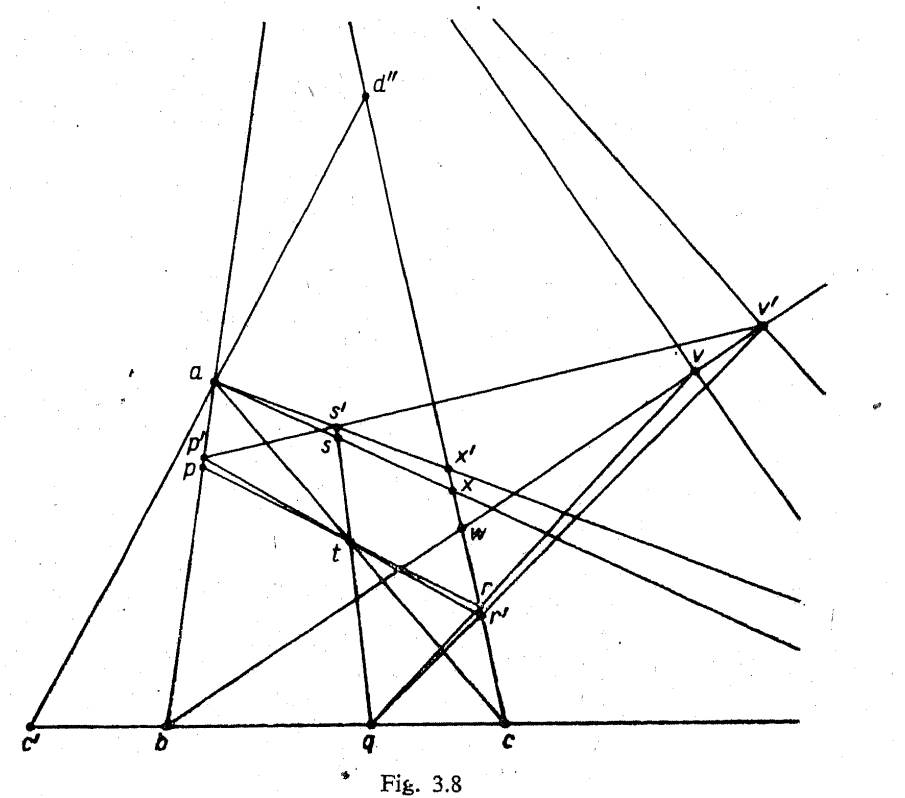

THEOREM 3.9. If $\mathfrak{Q}$ is a model of $\mathbf{G A}_{2}$ then $\mathfrak{F}_{\mathfrak{I}}$ is an ordered real-closed field and there is a point set $S$ convex in $\mathfrak{N}_{2}\left(\mathfrak{F}_{\mathfrak{g}}\right)$ such that $\mathfrak{Q}$ is isomorphic to $\mathfrak{A}_{2}\left(\mathfrak{\mho}_{\mathfrak{R}}, S\right)$.

The converse of this theorem fails unless we restrict ourselves to fields $\widetilde{F}$ which are isomorphic to the field of real numbers. This is seen from the following.

THEOREM 3.10. (i) Every $S$-restricted affine space over $\mathfrak{R}$ is a model of $\mathbf{G A}_{2}$ provided the point set $S$ is convex in $\mathfrak{H}_{2}(\mathfrak{R})$.

(ii) If a real-closed ordered field $\mathfrak{\&}$ is not isomorphic to $\mathfrak{R}$, then there is a set $S$ convex in $\mathfrak{R}_{2}(\widetilde{J})$ and such that $\mathfrak{U}_{2}(\mathfrak{F}, S)$ is not a model of $\mathbf{G A}_{2}$.

Proof. The proof of Theorem 3.10 (i) is straightforward. To prove part (ii) notice that in such a real-closed field $\tilde{F}$ there is a non-empty subset $Y$ of $F$ which is bounded from below but has no greatest lower bound. Now if $S$ is the set of all points $a=\left\langle x_{1}, x_{2}\right\rangle \in F \times F$ where $x_{1}$ is a lower bound of $Y$, then $S$ is open and convex, but the axiom $\mathbf{A}_{9}^{\Phi \Psi}$ where

$$
\begin{aligned}
& \Phi=\exists t[B(p q r) \wedge B(p a s) \wedge B(q a t) \wedge B(r s t)], \\
& \Psi=\forall t\left[B(p q r) \wedge B(p a s) \wedge B(q b t) \rightarrow \neg B\left(r s^{\prime} t\right)\right]
\end{aligned}
$$

is false.

Theorems 3.9 and 3.10 do not yield a solution of the representation problem for $\mathbf{G A}_{2}$. A satisfactory solution would consist in providing a purely mathematical (and sufficiently simple) characterization of the class of those restricted affine spaces over an arbitrary real closed ordered field which are models of $\mathbf{G A}_{2}$.

Remark 3.11. Let $\mathscr{K}$ and $\mathscr{L}$ be classes of relational systems $\mathfrak{H}_{2}(\mathfrak{F}, S)$ where $S$ is any point set convex in $\mathfrak{R}_{2}(\mathfrak{F}), \mathfrak{F}$ being any real closed field in case of $\mathscr{K}$, and $\mathfrak{F}$ is the field of real numbers in case of $\mathscr{L}$. By Theorems 3.9 and $3.10(\mathrm{ii}), \mathrm{T}(\mathscr{K})$ is a subtheory of $\mathbf{G A}_{2}$. An axiom system for $\mathbf{T}(\mathscr{K})$ may be provided by replacing the continuity axiom. schema by the sentence $S$ (see 3.1) and the sequence of axioms

$$
A_{10}^{n^{\prime}}=\llbracket\left\{L\left(v_{1} v_{2} v_{3}\right) \wedge B\left(v_{0} v_{1} v_{2}\right) \wedge \neg v_{0}=v_{1} \wedge \neg v_{1}=v_{2} \rightarrow \mathrm{HF}\left(R^{n}\right)\right\} \rrbracket
$$

for $n=2$ and for all odd numbers $n$, where

$$
\boldsymbol{R}^{2}=\llbracket \neg v_{1}^{2} \leqslant \mathbf{4} \cdot v_{0} \cdot v_{2} \rightarrow \exists v_{3}\left(v_{0}+v_{3} \cdot v_{1}+v_{3}^{2} \cdot v_{2}=0\right) \rrbracket
$$

and for $n>2$

$$
\boldsymbol{R}^{n}=\llbracket \neg v_{n}=\mathbf{0} \rightarrow \exists v_{n+1}\left(v_{0}+v_{n+1} \cdot v_{1}+v_{n+1}^{2} \cdot v_{2}+\ldots+v_{n+1}^{n} \cdot v_{n}=0\right) \rrbracket
$$

(cf. Lemma 2.19 and Tarski [15]. In these two formulas the expressions of the form $v_{i}^{j}$ (for arbitrary positive integers $i, j$ ) is defined by recursion on $j: \boldsymbol{v}_{i}^{\prime}=\boldsymbol{v} i$ and $v_{i}^{j+1}=v_{i} \cdot v_{i}^{j}$; in the first formula 4 is of course an abbreviation for $1+1+1$ +1. In Prestel-Szczerba [7] it is shown that $\mathbf{T}(\mathscr{L})$ is not (recursively) axiomatizable. Hence, in view of 3.10 (i), it clearly follows that $\mathbf{T}(\mathscr{L})$ is a proper extension of $\mathbf{G A}_{2}$.

Remark 3.12. Consider the affine geometry $\mathbf{G A}_{2}^{*}$, formulated in second-order language, whose axiom system is obtained from that of $\mathbf{G A}_{2}$ by replacing all the axioms $\mathbf{A}_{9}^{\phi \Psi}$ by the non-elementary continuity axiom

$$
\forall X Y\{\exists a \forall x \in X \forall y \in Y B(a x y) \rightarrow \exists b \forall x \in X \forall y \in Y B(x b y)\} .
$$

From Theorems 3.9 and 3.10 (i) we easily conclude that the class of models of $\mathbf{G A}_{2}^{*}$ 政 $(\mathfrak{R}, S)$, where $S$ ranges over all is the class of relational systems isomorphic to $\mathfrak{A}_{2}(\mathfrak{R}, S)$, where $S$ rang theory of $\mathscr{L}$ coincides with $\mathbf{G A}_{2}^{*}$.

§ 4. Metamathematical properties of $\mathbf{G A}_{2}$. We now turn to the problem of completeness, decidability, and finite axiomatizability of $\mathbf{G A}_{2}$. Let $\mathfrak{A}=\langle A, B\rangle$ be a model of $\mathbf{G A}_{2}$. By Theorem 3.9 we may assume that $\mathfrak{Q}=\mathfrak{Q}_{2}(\mathfrak{F}, S)$ for a certain ordered, real-closed field $\mathbb{F}$ and a set $S$ convex in $\mathfrak{Q}_{2}(\mathfrak{F})$.

DEFINITION 4.1. Let

$$
\begin{aligned}
& \Uparrow=\exists a b[B(a x y) \wedge B(x y b) \wedge B(a z u) \wedge B(z u b)] \vee \\
& \vee\{\forall a[B(x y a) \rightarrow \neg B(z u a)] \wedge \forall b[\neg b=y \wedge B(y b u) \rightarrow \exists c B(x b c) \wedge B(z u c)]\} .
\end{aligned}
$$

Moreover, let

$$
a b \uparrow c d \quad \text { iff } \quad \mathfrak{A} \vDash \mathbb{1}\left[\begin{array}{llll}
x & y & z & u \\
a & b & c & d
\end{array}\right]
$$


Instead of $\uparrow \uparrow\left(v_{0} v_{1} v_{2} v_{3}\right)$ we shall often write $v_{0} v_{1} \uparrow \uparrow v_{2} v_{3}$.

The relation $\uparrow$ is a hyperbolic type of parallelity of halflines. In Fig. 4.2 we have $a b \uparrow c d$ but neither $a b \uparrow c^{\prime} d^{\prime}$ nor $a b \uparrow c^{\prime \prime} d^{\prime \prime}$. As in the case of hyperbolic geometry, one can prove that the relation $\Uparrow$ is reflexive, symmetric and transitive.

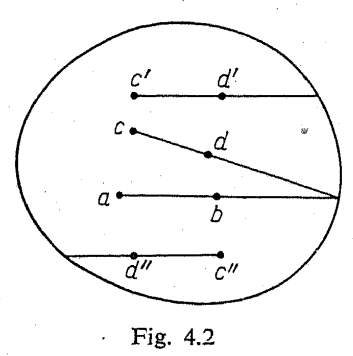

LEMMA 4.3.

(i) $a b$ 1 $a b$,

(ii) $a b \uparrow c d$ whenever $c d \uparrow a b$,

(iii) if $a b \uparrow p q$ and $c d \uparrow p q$ and $p \neq q$ then $a b \uparrow c d$.

Definttion 4.4. The collection of all non-trivial pairs of points (i.e. pairs $\langle a, b\rangle$ where $a \neq b$ ) may be partitioned into equivalence classes with respect to $\uparrow$. For convenience, we enrich each class by all trivial pairs. Any such enriched class will be called a direction.

Definition 4.5. The direction $\vartheta$ is a vertex if all pairs $\langle x, y\rangle \in \vartheta$ satisfy the formula

\section{$V=\forall a \forall b \exists c \forall d \forall e[B(a x b) \wedge B(a y d) \wedge B(b y e) \wedge B(x y c) \wedge$}

(see Fig. 4.6).

$$
\wedge \neg a=x \wedge \neg b=x \rightarrow \neg B(d c e)]
$$

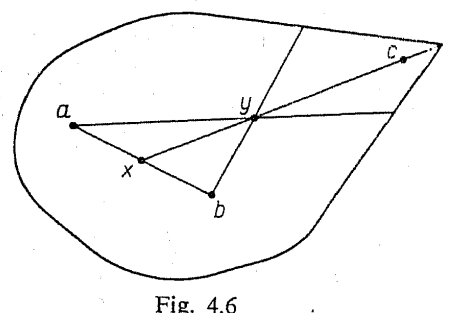
It is easy to see that if one non-degenerate pair $\langle x, y\rangle \in \vartheta$ satisfies the formula $V$,
then $\vartheta$ is a vertex.
Defintrion 4.7. We say that a point $a$ is definable in a model $\mathfrak{A}$ if there is a formula $\Phi$, with one free variable $x$, such that

$$
p=a \quad \text { iff } \quad \mathfrak{A} \vDash \Phi\left[\begin{array}{l}
x \\
p
\end{array}\right]
$$

Analogously we shall understand the definability of directions, vertices atc.

LeMmA 4.8. Let $\mathfrak{Q}=\mathfrak{U}_{2}(\mathfrak{R}, S)$, where $S$ is a convex set in $\mathfrak{U}_{2}(\mathfrak{R})$. If in $\mathfrak{Q}$ there are three different definable points $a, b, c$, such that $B_{\mathfrak{M}}(a b c)$, then the set of points definable in $\mathfrak{Q}$ is dense in the segment $[a, b]$.

Pro of. Without loss of generality, we may assume that $a=\langle x, 0\rangle, b=\langle y, 0\rangle$, $c=\langle 0,0\rangle$, and $x\rangle y\rangle 0$. Assume, moreover, that two points $p_{u}=\langle u, 0\rangle$ and $p_{v}=\langle v, 0\rangle$ are definable in $\mathfrak{R}$. Let $a$ be definable by a formula $\boldsymbol{P}_{a}, b$ by $\boldsymbol{P}_{b}, c$ by $\boldsymbol{P}_{c}, p_{u}$ by $\boldsymbol{P}$ and $p_{v}$ by $\boldsymbol{Q}$. Let

$$
f(u, v)=2 \frac{u v}{u+v} \quad \text { for } \quad u+v \neq 0 .
$$

The point

$$
p_{f(u, v)}=\langle f(u, v), 0\rangle=\left\langle 2 \frac{u v}{u+v}, 0\right\rangle, \quad \text { where } \quad u+v \neq 0,
$$

is definable by the formula

$W=\exists a \exists b \exists c \exists p \exists q \exists r \exists s \exists t \exists v[\neg L(a b r) \wedge \sqcap L(a b v) \wedge$

$\wedge B(r a s) \wedge L(r t p) \wedge L(s v p) \wedge L(s q t) \wedge L(r q v) \wedge B(t x v) \wedge$

$$
\left.\wedge B(p x q) \wedge P_{a}(a) \wedge P_{b}(b) \wedge P_{c}(c) \wedge P(p) \wedge Q(q)\right]
$$

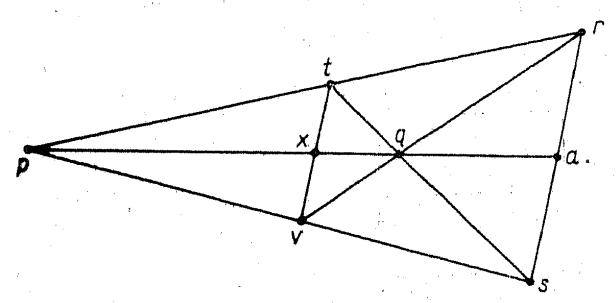

Fig. 4.9

(see Fig. 4.9). Thus, it will be sufficient to prove that the set $D$, containing numbers $x$ and $y$ and closed under the operation $f$, is dense in the segment $[x, y]$. The function $g$ defined by

$$
g(u)=\frac{y(x-u)}{u(x-y)}
$$


is a one-to-one monotone mapping of the segment $[x, y]$ onto the segment $[0,1]$. This mapping transforms the operation $f$ into the operation $f^{\prime \prime}$, where $f^{\prime}(u \cdot v)=\frac{1}{2}(u+v)$. Therefore $D^{\prime}$, the image of $D$, is the set diadic numbers of the segment $[0,1]$. Since $D^{\prime}$ is dense in $[0,1]$ and $g$ is a monotone function, $D$ is also dense in $[x, y]$. This proves the lemma.

THEOREM 4.10. The theory $\mathbf{G A}_{2}$ is incomplete and has in fact $2^{\mathrm{N}_{0}}$ complete extensions.

Proof. For any given real $x>0$ let $S_{x}$ be the interior of the pentagon in $\mathfrak{H}_{2}(\mathfrak{R})$ with the vertices $\langle 2,-1\rangle,\langle 0,-1\rangle,\langle 0,1\rangle,\langle 2,1\rangle$ and $\langle 4+x, 0\rangle$. By $3.10(\mathrm{i})$ $\mathbf{T}\left(\mathfrak{N}_{2}\left(\mathfrak{R}, S_{x}\right)\right)$ is an extension of $\mathbf{G A}_{2}$ for any $x>0$. We show that $\mathbf{T}\left(\mathfrak{H}_{2}\left(\mathfrak{R}, S_{x}\right)\right)$ $\neq \mathbf{T}\left(\mathfrak{U}_{2}\left(\mathfrak{R}, S_{y}\right)\right)$ for any real $x, y$ with $0<x<y<8$. Let $x \geqslant 0$.

(1) The point $\langle 1,0\rangle$ is defined in $\mathfrak{N}_{2}\left(\mathfrak{R}, S_{x}\right)$ by the formula

$$
P_{\langle 1,0\rangle}=\exists p \exists q[\neg L(p q z) \wedge V(z p) \wedge V(p z) \wedge V(z q) \wedge V(q z)]
$$

(see Fig. 4.11).

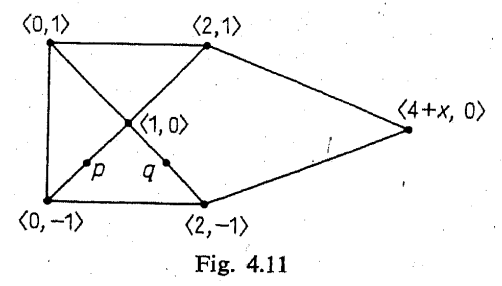

(2) The point $\langle 2,0\rangle$ is defined in $\mathfrak{N}_{2}\left(\mathfrak{R}, S_{x}\right)$ by the formula

$$
P_{\langle 2,0\rangle}=\exists p \exists q\left[P_{\langle 1,0\rangle}(p) \wedge \neg V(z p) \wedge V(p z) \wedge V(z q) \wedge V(q z) \wedge \neg z=p\right]
$$

(see Fig. 4.12).

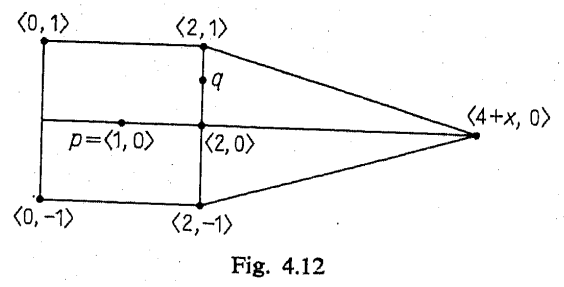

(3) The point $\langle 4,0\rangle$ is defined in $\mathfrak{N}_{2}\left(\Re, S_{x}\right)$ by the formula

$P_{\langle 4,0\rangle}=\exists p \exists q \exists r \exists s \exists t \exists u\left[P_{\langle 1,0\rangle}(t) \wedge P_{\langle 2,0\rangle}(u) \wedge \neg L(t u p) \wedge V(u p) \wedge V(u s) \wedge V(t r) \wedge\right.$ $\wedge V(q p) \wedge p q$ \} $u t \wedge t p \uparrow u r \wedge t r \Uparrow u s \wedge z s \uparrow u p \wedge B(p r s) \wedge B(t u z)]$.

(see Fig. 4.13).

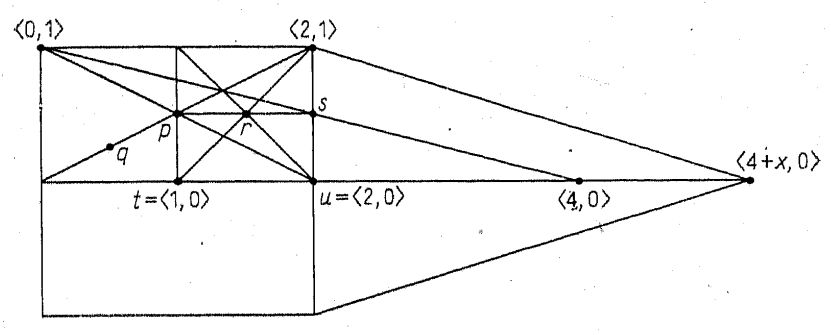

Fig. 4.13 .

(4) The point $\langle 2+x / 3,0\rangle$ is defined in $\mathfrak{N}_{2}\left(\mathfrak{R}, S_{\boldsymbol{x}}\right)$ by the formula $P_{\langle 2+x / 3,0\rangle}=\exists p \exists q \exists r \exists s \exists t \exists u \exists v\left[P_{\langle 1,0\rangle}(t) \wedge P_{\langle 2,0\rangle}(u) \wedge P_{\langle 4,0\rangle}(v) \wedge\right.$ $\wedge \neg L(t u r) \wedge B(r u s) \wedge V(u r) \wedge V(r u) \wedge V(z s) \wedge V(u q) \wedge V(p q) \wedge$

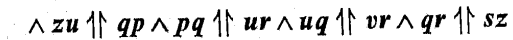

(see Fig. 4.14).

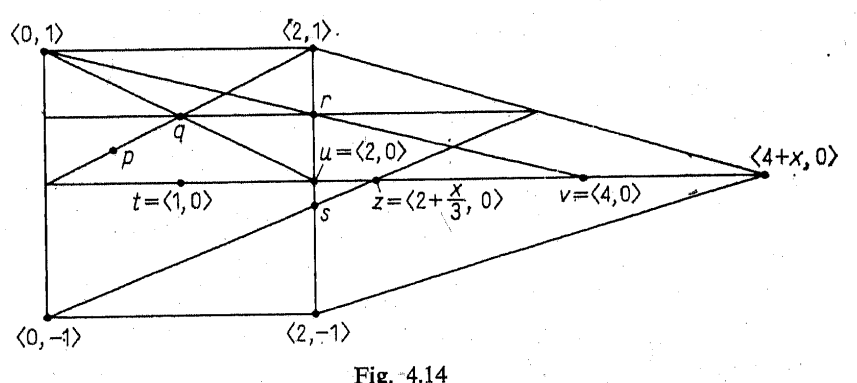

By 4.8 the set of definable points is dense in the segment $[\langle 2,0\rangle,\langle 4,0\rangle]$. Hence there is a number $w$ such that the point $\langle 2+w, 0\rangle$ is definable and separates points $\langle 2+x / 3,0\rangle$ and $\langle 2+y / 3,0\rangle$. Let $\boldsymbol{P}_{\langle 2+w, 0\rangle}$ be a formula having $\boldsymbol{v}_{0}$ as the only free variable and defining the point $\langle 2+w, 0\rangle$ in $\mathfrak{Q}_{2}\left(\mathfrak{R}, S_{x}\right)$. The sentence

$$
\exists p \exists q \exists r\left[P_{\langle 1,0\rangle}(p) \wedge P_{\left\langle 2+x_{i} 3,0\right\rangle}(q) \wedge P_{\langle 2+w, 0\rangle}(r) \wedge B(p q r)\right]
$$

is true in $\mathfrak{2}_{2}\left(\mathfrak{R}, S_{x}\right)$ but not $\mathfrak{N}_{2}\left(\mathfrak{R}, S_{y}\right)$. This completes the proof.

THEOREM 4.15. The theory $\mathbf{G A}_{2}$ and all of its subtheories are undecidable (in other words, $\mathbf{G A}_{2}$ is hereditarily undecidable).

Proof We construct the restricted affine plane $\mathfrak{I}=\mathfrak{A}_{2}(\mathfrak{R}, S)$ by taking for $S$ the interior of the smallest convex set which contains the points

$$
\begin{gathered}
a=\langle 1,0\rangle, \quad b=\langle(1+\sqrt{3}) / 2 \sqrt{2},(1-\sqrt{3}) / 2 \sqrt{2}\rangle, \quad c=\langle 0,-1\rangle, \\
d=\langle-1,0\rangle, \quad e=\langle(1-\sqrt{3}) / 2 \sqrt{2},(1+\sqrt{3}) / 2 \sqrt{2}\rangle,
\end{gathered}
$$


and $f_{n}=\left\langle n / \sqrt{n^{2}+1}, 1 / \sqrt{n^{2}+1}\right\rangle$ for $n=0,1,2, \ldots$ (see Fig. 4.16). By Theorem 3.10 (i) $\mathfrak{A}$ is a model of $\mathbf{G A}_{2}$. From Fig. 4.16 it is seen that in this mode the vertex $a$ can be singled out as the unique accumulation point of the set of vertices.

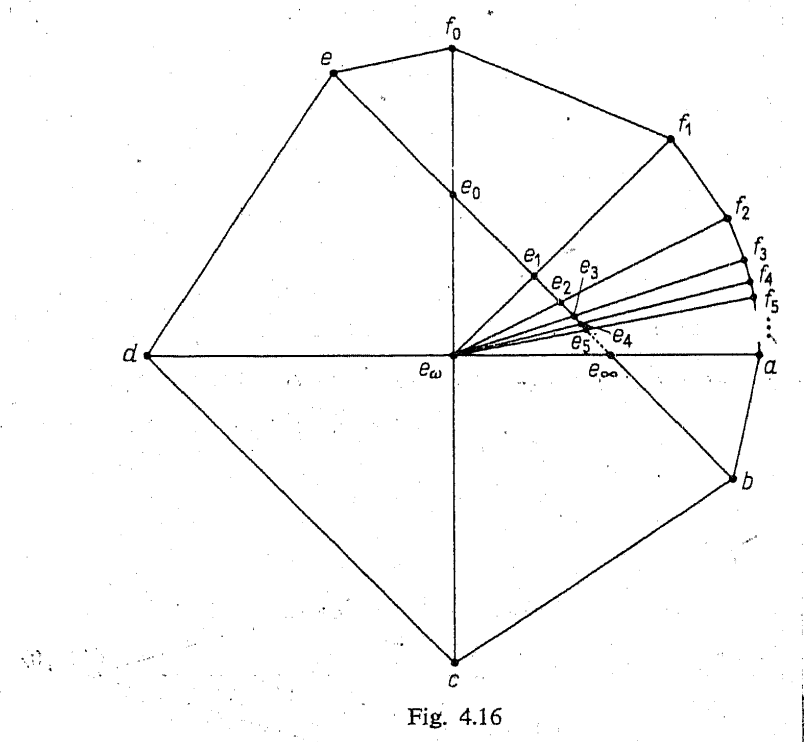

On the other hand, $b$ can be characterized as the only vertex adjacent to $a$ (i.e. such that there are no vertices between $a$ and $b$ ). Analogously $c$ can be defined as the only vertex different from $a$ and adjacent to $b$; the vertices $d, e, f_{0}, f_{1}$ are, in turn, defined successively in a similar manner. It is obvious that $a, \ldots, f_{1}$ are definable in $\mathfrak{A}$ by means of formulas in $\mathbf{L}(\boldsymbol{B})$. Hence we can define in $\mathfrak{A}$ the points $e_{0}=\langle 0,1 / \sqrt{2}\rangle$, $e_{1}=\langle 1 / 2 \sqrt{2}, 1 / 2 \sqrt{2}\rangle, e_{\infty}=\langle 1 / \sqrt{2}, 0\rangle$ and $e_{\omega}=\langle 0,0\rangle$, and therefore the algebra $\mathfrak{I}_{\mathfrak{Q}}$ with the segment $\left[e_{0}, e_{\infty}\right)$ as its universe. The algebra $\mathfrak{H}_{\mathscr{N}}$ is isomorphic to the field $\mathfrak{R}$ restricted to its positive cone $R^{+}$. We define the set $N$ of points $e_{0}, e_{1}, e_{2}, \ldots$, by stipulating that $p \in N$ if and only if $B_{9}\left(e_{0} p e_{\infty}\right), p \neq e_{\infty}$ and the pair $\left\langle e_{\omega} ; p\right\rangle$ belongs to a vertex. It is easy to check by analytical methods that $\mathfrak{I}_{\mathfrak{Q}}$, restricted to the set $N$, is isomorphic to the standard model of the arithmetic of natural numbers. Thus the theory $\mathbf{Q}$ of Tarski-Mostowski-Robinson [17], $\mathrm{p}_{i} 51$ is relatively interpretable in $\mathbf{T}(\mathfrak{V})$ and therefore, by Theorem 8 of Tarski-Mostowski-Robinson [17], $\mathbf{T}(\mathfrak{2})$ is hereditarily undecidable. Since $\mathbf{G A}_{2}$ is a subtheory of $\mathbf{T}(\mathfrak{2}), \mathbf{G A}_{2}$ is also hereditarily undecidable.

Theorems 4.10 and 4.15 , when compared with the corresponding results for $\mathbf{A}_{2}$ in Szmielew [14] (Theorems 3.2. and 3.3 for $n=2$, pp. $50 \mathrm{ff}$.), demonstrate that there are fundamental metamathematical differences between the theories $\mathbf{A}_{2}$ and $\mathbf{G A}_{2}$ despite the fact that the axiom systems of these two theories have been constructed by essentially the same method.

THEOREM 4.16. The theory $\mathbf{G A}_{2}$ is not finilely axiomatizable.

Proof. In Tarski [16] a finitely axiomatizable theory $\mathbf{E}_{2}^{\prime \prime}$ was introduced. Any model of $\mathbf{E}_{2}^{\prime \prime}$ was shown to be (up to isomorphism) the Cartesian plane over an ordered commutative field $\mathfrak{F}$ (in symbols $\mathfrak{C}_{2}(\mathfrak{F})$ ), i. e., an expansion of $\mathfrak{V}_{2}(\mathfrak{F})$ obtained by including a quaternary relation $D_{\Re}^{(2)}$ defined as follows:

$$
D_{\Im}(a b c d) \quad \text { iff } \quad\left(a_{1}-b_{1}\right)^{2}+\left(a_{2}-b_{2}\right)^{2}=\left(c_{1}-d_{1}\right)^{2}+\left(c_{2}-d_{2}\right)^{2}
$$

where $d=\left\langle a_{1}, a_{2}\right\rangle, b=\left\langle b_{1}, b_{2}\right\rangle, c=\left\langle c_{1}, c_{2}\right\rangle$ and $d=\left\langle d_{1}, d_{2}\right\rangle$. Let us consider the theory $\mathbf{E}_{2}^{\prime}$ which is an extension of $\mathbf{G A}_{2}$ obtained by enriching the axiom system of $\mathbf{G A}_{2}$ by all the axioms of $\mathbf{E}_{2}^{\prime \prime}$. Since $\mathbf{E}_{2}^{\prime}$ is an extension of $\mathbf{E}_{2}^{\prime \prime}$, any model $\mathbf{E}_{2}^{\prime}$ must be isomorphic to $\mathfrak{C}_{2}(\mathfrak{F})$ for a certain ordered, commutative field $\mathfrak{F}$. Consider the reduct $\mathfrak{A}$ of $\mathfrak{C}_{2}(\mathfrak{F})$ by the relation $D_{\mathfrak{F}}$. The relational system $\mathfrak{A}$ is a model of $\mathbf{G A}_{2}$. Therefore (cf. 3.1 and 2.19) $\mathfrak{F}$ is a real-closed, ordered field, and hence $\mathfrak{C}_{2}(\mathfrak{F}$ ) is a model of elementary Euclidean geometry $\mathbf{E}_{2}$ (see Tarski [16], Theorem 1). Since any model of $\mathbf{E}_{2}$ is clearly a model of $\mathbf{E}_{2}^{\prime}$, we may conclude that $\mathbf{E}_{2}=\mathbf{E}_{2}^{\prime}$. It follows that $\mathbf{E}_{2}$ is a finite extension of $\mathbf{G A}_{2}$. Since $\mathbf{E}_{2}$ is not finitely axiomatizable (see Tarski [16], Theorem 4), neither is $\mathbf{G A}_{2}$.

It should be pointed out that we do not know any consistent extension of $\mathbf{G A}_{2}$ which is finitely axiomatizable; the problem whether such an extension exists is open.

Some further metamathematical results, of more special character, will be established in Section 6 (Theorems 6.12, 6.13, 6.14).

§ 5. Uniformly definable extensions of $\mathbf{G A}_{2}$. Let $\mathfrak{F}=\langle F, 0,1,+, \cdot, \leqslant\rangle$ be any real-closed ordered field, and let $S \subseteq F \times F$ be a convex set in $\mathfrak{A}_{2}(\mathfrak{F})$. Suppose $\mathfrak{A}=\mathfrak{Q}_{2}(\mathfrak{F}, S)$ is a model of $\mathbf{G A}_{2}$.

Definition 5.1. Consider the algebras $\mathfrak{H}_{\mathfrak{I}}$ and $\mathfrak{F}_{\mathfrak{M}}=\left\langle F_{\mathfrak{M}}, 0,1,+, \cdot, \leqslant\right\rangle$ defined in Section 2. Elements of $\mathfrak{S}_{\mathfrak{Q}}$ are points of the segment $\left[e_{0}, e_{\infty}\right)$ and elements of $\mathfrak{F}_{\mathfrak{Q}}$ are equivalence classes of pairs of such points. Let $f$ be the function mapping $\mathfrak{F}$ onto $\mathfrak{F}_{\mathfrak{I t}}$ and satisfying the conditions:

$$
\begin{array}{ll}
f(x)=\left\langle\left\langle\frac{x}{1+x}, 0\right\rangle,\langle 0,0\rangle\right\rangle_{I_{\approx}} & \text { whenever } x \geqslant 0, \\
f(x)=\left\langle\langle 0,0\rangle,\left\langle\frac{-x}{1-x} \cdot 0\right\rangle\right\rangle_{I_{\approx}} & \text { whenever } x<0 .
\end{array}
$$

Further, let $g$ be a function mapping $S$ into $F_{\mathfrak{Q P}} \times F_{\mathfrak{Q}}$ and such that $\quad \therefore$

$$
g(\langle x, y\rangle)=\langle f(x), f(y)\rangle \quad \text { for any }\langle x, y\rangle \in S .
$$


Remark 5.2. It is easy to see that $f$ is an isomorphism of $\mathfrak{F}$ into $\mathfrak{F}_{\mathfrak{A}}$ and $g$ is an isomorphism of $\mathfrak{Q}$ into $\mathfrak{A}_{2}(\mathfrak{F})$.

Definition 5.3. Let $K$ be the quinary relation between points of $S$ defined as follows:

$$
K\left(a x_{0} y_{0} x_{1} y_{1}\right) \quad \text { iff } \quad g(a)=\left\langle\left.\left\langle x_{0}, y_{0}\right\rangle\right|_{\approx},\left\langle x_{1}, y_{1}\right\rangle / \approx\right\rangle .
$$

We shall show that this relation is definable by means of a formula in $\mathbf{L}(\boldsymbol{B})$. (On this and the next two pages we use the term "definable" in the sense of "parametrically definable"; an analogous remark applies to related terms such as "definition", "defined", etc.) We start with the particular case where points $e_{0}=\langle 0,0\rangle, e_{\infty}$ $=\langle 1,0\rangle, e_{\omega}=\langle 0,1\rangle$ and $e^{\prime}=\langle 1,-1\rangle$ belong to $S$. Since $S$ is convex, the point $e_{1}=\left\langle\frac{1}{2}, 0\right\rangle$ also belongs to $S$.

First we shall prove that the relation $K$ is definable for any point $a$ belonging to the segment $\left[e_{0}, e_{\infty}\right)$. The definition is in fact a natural isomorphism from $\mathfrak{H}_{\mathfrak{2}}$ into $\mathfrak{F}$, expressed in the language $\mathbf{L}(\boldsymbol{B})$ by means of Lemma 2.14. Let

$$
\begin{aligned}
K_{1}=\left[c=v_{12} \wedge v_{8}\right. & \left.=v_{9} \wedge H\left(v_{2}=v_{3}+1\right)\right] \vee \\
& \vee\left[c \neq v_{12} \wedge v_{10}=v_{11} \wedge H\left(v_{0} \cdot v_{4}+v_{0}=v_{1} \cdot v_{4}+v_{1}+v_{4}\right)\right] .
\end{aligned}
$$

For any $a \in\left[e_{0}, e_{\infty}\right]$ we have $\boldsymbol{g}(a)=\left\langle\left\langle x_{0}, y_{0}\right\rangle / \approx,\left\langle x_{1}, x_{1}\right\rangle / \approx\right\rangle \quad$ iff $\quad \mathfrak{A} \vDash \boldsymbol{K}_{1}\left[\begin{array}{lllllllll}\boldsymbol{a} & \boldsymbol{b} & \boldsymbol{c} & \boldsymbol{d} & \boldsymbol{v}_{8} & \boldsymbol{v}_{9} & \boldsymbol{v}_{10} & \boldsymbol{v}_{11} & \boldsymbol{v}_{12} \\ e_{0} & e_{1} & e_{\infty} & \boldsymbol{e}_{\omega} & x_{0} & y_{0} & x_{1} & y_{1} & a\end{array}\right]$.

The relation $K$ is also definable for $a$ from the segment $\left[e_{0} e_{\omega}\right]$ by the formula

$$
K_{2}=\exists v_{14} \exists v_{15} \exists v_{16} \exists v_{17} \exists v_{18}\left[B\left(a v_{18} d\right) \wedge B\left(v_{12} v_{18} e\right) \wedge\right.
$$

$$
\left.\wedge K_{1}\left(\begin{array}{lllll}
v_{8} & v_{9} & v_{10} & v_{11} & v_{12} \\
v_{14} & v_{15} & v_{16} & v_{17} & v_{18}
\end{array}\right) \wedge H F\left(v_{0}=0 \wedge v_{1}=v_{3}+v_{1} \cdot v_{3}\right)\right]
$$

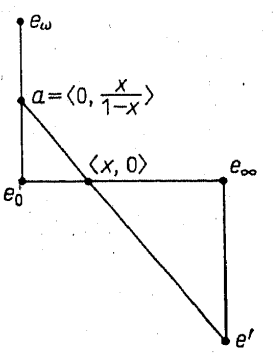

Fig. 5.4

(see Fig. 5.4), i.e., for any $a \in\left[e_{0} e_{\omega}\right]$ we have

$$
g(a)=\left\langle\left\langle x_{0}, y_{0}\right\rangle / \approx,\left\langle x_{1}, y_{1}\right\rangle / \approx\right\rangle
$$

$$
\mathfrak{A} \vDash \boldsymbol{K}_{2}\left[\begin{array}{llllllllll}
\boldsymbol{a} & \boldsymbol{b} & \boldsymbol{c} & \boldsymbol{d} & \boldsymbol{e} & \boldsymbol{v}_{\mathbf{8}} & \boldsymbol{v}_{9} & \boldsymbol{v}_{10} & \boldsymbol{v}_{11} & \boldsymbol{v}_{12} \\
e_{0} & e_{1} & e_{\infty} & e_{\omega} & e^{\prime} & x_{0} & y_{0} & x_{1} & y_{1} & a
\end{array}\right]
$$

Similarly for $a \in\left[e_{\infty} e_{0,}\right]$ the formula

$$
\begin{aligned}
K_{3}= & \exists v_{14} \exists v_{15} \exists v_{16} \exists v_{17} \exists v_{18}\left[B\left(a v_{18} d\right) \wedge B\left(v_{12} v_{18} e\right) \wedge\right. \\
& \wedge K_{1}\left(\begin{array}{lllll}
v_{8} & v_{9} & v_{10} & v_{11} & v_{12} \\
v_{1.4} & v_{15} & v_{16} & v_{17} & v_{18}
\end{array}\right) \wedge \mathrm{HF}\left(v_{0} \cdot v_{3}+v_{3}+v_{3}=1 \wedge v_{1} \cdot v_{3}+1=v_{3}\right)
\end{aligned}
$$

defines the relation $A$ : for any $a \in\left[e_{\infty} e_{\omega}\right]$ we have

$$
g(a)=\left\langle\left\langle x_{0}, y_{0}\right\rangle / \approx,\left\langle x_{1}, y_{1}\right\rangle \mid \approx\right\rangle
$$

iff

$$
\mathfrak{A} \vDash \boldsymbol{K}_{3}\left[\begin{array}{llllllllll}
\boldsymbol{a} & \boldsymbol{b} & \boldsymbol{c} & \boldsymbol{d} & \boldsymbol{e} & \boldsymbol{v}_{8} & \boldsymbol{v}_{9} & \boldsymbol{v}_{10} & \boldsymbol{v}_{11} & \boldsymbol{v}_{12} \\
e_{0} & e_{1} & e_{\infty} & e_{\omega} & e^{\prime} & x_{0} & y_{0} & x_{1} & y_{1} & a
\end{array}\right]
$$

Now we may define the relation $K$ for all points belonging to the set $T$, the boundary of the triangle $e_{0}, e_{\infty}, e_{\omega}$. The set $T$ is determined by the stipulation

$$
\text { for every } a \text { in } \mathfrak{N}, \quad a \in T \quad \text { iff } \quad \mathfrak{N} \vDash T\left[\begin{array}{lllll}
\boldsymbol{a} & \boldsymbol{b} & \boldsymbol{c} & \boldsymbol{d} & \boldsymbol{x} \\
e_{0} & e_{1} & e_{\infty} & e_{\omega} & a
\end{array}\right] \text {, }
$$

where $\boldsymbol{T}$ is the formula $\boldsymbol{B}(\boldsymbol{a x c}) \vee \boldsymbol{B}(\boldsymbol{a x d}) \vee \boldsymbol{B}(\boldsymbol{c x d})$. For $a \in T$ the relation $\boldsymbol{K}$ is defined by the formula

$$
K_{4}=\left[B\left(a v_{12} c\right) \wedge K_{1}\right] \vee\left[B\left(a v_{12} d\right) \dot{\wedge} K_{2}\right] \vee\left[B\left(c v_{12} d\right) \wedge K_{3}\right],
$$

i.e., for any $a \in T$ we have

iff

$$
g(a)=\left\langle\left.\left\langle x_{0}, y_{0}\right\rangle\right|_{\approx},\left\langle x_{1}, y_{1}\right\rangle \mid \approx\right\rangle
$$

$$
\mathfrak{A} \vDash K_{4}\left[\begin{array}{llllllllll}
a & b & c & d & e & v_{8} & v_{9} & v_{10} & v_{11} & v_{12} \\
e_{0} & e_{1} & e_{\infty} & e_{\omega} & e^{\prime} & x_{0} & y_{0} & x_{1} & y_{1} & a
\end{array}\right]
$$

Now we may extend the definition of the relation $K$ to the entire set $S$ (see Fig. 5.5).

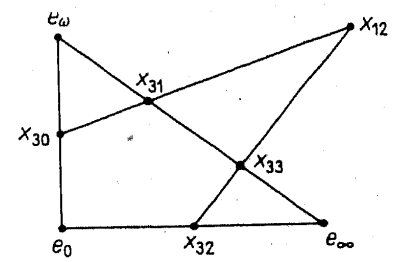

Fig. 5.5 
The formula

$K_{5}=\exists v_{14} \exists v_{15} \ldots \exists v_{33}\left[T\left(v_{30}\right) \wedge T\left(v_{31}\right) \wedge T\left(v_{32}\right) \wedge T\left(v_{33}\right) \wedge\right.$

$$
\begin{aligned}
& \wedge K_{4}\left(\begin{array}{lllll}
v_{8} & v_{9} & v_{10} & v_{11} & v_{12} \\
v_{15} & v_{15} & v_{16} & v_{17} & v_{30}
\end{array}\right) \wedge K_{4}\left(\begin{array}{lllll}
v_{8} & v_{9} & v_{10} & v_{11} & v_{12} \\
v_{18} & v_{19} & v_{20} & v_{21} & v_{31}
\end{array}\right) \wedge \\
& \wedge K_{4}\left(\begin{array}{lllll}
v_{8} & v_{9} & v_{10} & v_{11} & v_{12} \\
v_{22} & v_{23} & v_{24} & v_{25} & v_{32}
\end{array}\right) \wedge K_{4}\left(\begin{array}{lllll}
v_{8} & v_{9} & v_{10} & v_{11} & v_{12} \\
v_{26} & v_{27} & v_{28} & v_{29} & v_{33}
\end{array}\right) \wedge \\
& \wedge L\left(v_{12} v_{30} v_{31}\right) \wedge L\left(v_{12} v_{32} v_{33}\right) \wedge \neg L\left(v_{30} v_{31} v_{32}\right) \wedge \\
& \wedge \mathbf{H F}\left(\exists v _ { 1 1 } \exists v _ { 1 2 } \exists v _ { 1 3 } \exists v _ { 1 5 } \exists v _ { 1 6 } \left[\neg \ddot{v}_{11} \cdot v_{11}+v_{12} \cdot v_{12}+v_{13} \cdot v_{13}=0 \wedge\right.\right. \\
& \wedge \neg v_{14} \cdot v_{14}+v_{15} \cdot v_{15}+v_{16} \cdot v_{16}=0 \wedge \\
& \wedge v_{0} \cdot v_{11}+v_{3} ; v_{12}+v_{5} \cdot v_{13}=0 \wedge \\
& \wedge v_{1} \cdot v_{11}+v_{4} \cdot v_{12}+v_{6} \cdot v_{13}=0 \wedge \\
& \wedge v_{0} \cdot v_{14}+v_{7} \cdot v_{15}+v_{9} \cdot v_{16}=0 \wedge \\
& \left.\left.\left.\wedge v_{1} \cdot v_{14}+v_{8} \cdot v_{15}+v_{10} \cdot v_{16}=0\right]\right)\right]
\end{aligned}
$$

defines the relation $K$, i.e., we have.

$$
g(a)=\left\langle\left\langle x_{0}, y_{0}\right\rangle / \approx,\left\langle x_{1}, y_{1}\right\rangle \mid \approx\right\rangle
$$

iff

$$
\mathfrak{A} \vDash \boldsymbol{K}_{5}\left[\begin{array}{llllllllll}
\boldsymbol{a} & \boldsymbol{b} & \boldsymbol{c} & \boldsymbol{d} & \boldsymbol{e} & \boldsymbol{v}_{8} & \boldsymbol{v}_{9} & \boldsymbol{v}_{10} & \boldsymbol{v}_{11} & \boldsymbol{v}_{12} \\
e_{0} & e_{1} & e_{\infty} & e_{\omega} & e^{\prime} & x_{0} & y_{0} & x_{1} & y_{1} & a
\end{array}\right] .
$$

In general, the points $\langle 0,0\rangle,\langle 1,0\rangle,\langle 0,1\rangle$ and $\langle 1,-1\rangle$ do not necessarily belong to the set $S$, but since $S$ is non-empty and open, there are $s, t, u$ in $F$ such that the points $e_{0}=\langle s, u\rangle, e_{\infty}=\langle s+t, u\rangle, e_{\omega}=\langle s, u-\mathrm{t}\rangle$ and $e^{\prime}=\langle s+t, u-t\rangle$ do belong to $S$. Since $S$ is convex, the point $e_{1}=\left\langle s+\frac{1}{2} t, u\right\rangle$ also belongs to $S$. Let $f(s)=\left\langle s_{1}, s_{2}\right\rangle \mid \approx$, $f(t)=\left\langle t_{1}, t_{2}\right\rangle / \approx$ and $f(u)=\left\langle u_{1}, u_{2}\right\rangle \mid \approx$. Obviously $s_{1}, s_{2}, t_{1}, t_{2}, u_{1}, u_{2}$ belong to $H=\left[e_{0}, e_{\infty}\right) \subseteq S$. Setting

$$
\left.\begin{array}{rl}
K=\exists v_{20} \exists v_{21} \exists v_{22} \exists v_{23}\left[K_{5}\left(\begin{array}{llll}
v_{8} & v_{9} & v_{10} & v_{11} \\
v_{20} & v_{21} & v_{22} & v_{23}
\end{array}\right) \wedge\right. \\
\wedge & \quad H F\left(v_{0}=v_{3}+v_{6} \cdot v_{4} \wedge v_{1}=v_{5}+v_{7} \cdot v_{4}\right)
\end{array}\right]
$$

We easily obtain

LEMMA 5.6. If $\mathfrak{N}$ is a model of $\mathbf{G A}_{2}$, there are points $e_{0}, e_{1}, e_{\infty}, e_{\omega v}, e^{\prime}$, $s_{1}, s_{2}, t_{1}, t_{2}, u_{1}, u_{2}$ such that for any points $a, x_{0}, x_{1}, y_{0}, y_{1}$ we have:

$$
g(a)=\left\langle\left\langle x_{0}, y_{0}\right\rangle\left|\approx,\left\langle x_{1}, y_{1}\right\rangle\right| \approx\right\rangle
$$

iff

$$
\mathfrak{A} \vDash \boldsymbol{K}\left[\begin{array}{llllllllllllllll}
\boldsymbol{v}_{0} & \boldsymbol{v}_{1} & \boldsymbol{v}_{2} & \boldsymbol{v}_{3} & \boldsymbol{v}_{4} & \boldsymbol{v}_{8} & \boldsymbol{v}_{9} & \boldsymbol{v}_{10} & \boldsymbol{v}_{11} & \boldsymbol{v}_{12} & \boldsymbol{v}_{14} & \boldsymbol{v}_{15} & \boldsymbol{v}_{16} & \boldsymbol{v}_{17} & \boldsymbol{v}_{18} & \boldsymbol{v}_{19} \\
e_{0} & e_{1} & e_{\infty} & e_{\omega} & e^{\prime} & x_{0} & y_{0} & x_{1} & y_{1} & a & s_{1} & s_{2} & t_{1} & t_{2} & u_{1} & u_{2}
\end{array}\right]
$$

We wish discuss some extensions of $\mathbf{G A}_{2}$. The most interesting examples of such extensions are defined model-theoretically as the elementary theories of some mathematically important classes of restricted affine spaces. We state below a fairly general result concerning extensions of this kind. To formulate this conveniently, we refer to the formalism of the elementary theories of ordered fields, i. e., to the language of the theory of ordered fields.

Defintrion 5.7. Let $\Phi$ and $\Psi$ be formulas in $\mathbf{L}\left(\mathbf{0}, \mathbf{1},+, \cdot, \leqslant>\right.$ and let $\mathbf{v}_{0}, \ldots, \mathbf{v}_{n}$ be a sequence without repetitions of all variables occurring free in $\Phi$ and $\exists v_{0} \exists v_{1} \Psi$. Given any ordered field $\mathfrak{F}=\langle F, 0,1,+, \cdot, \leqslant\rangle$, the formula $\Psi$ and sequence $v_{0}, \ldots, v_{n}$ of elements of $F$ define a subset of $F \times F$

$$
S_{\widetilde{F}}^{\Psi}\left(v_{0}, \ldots, v_{n}\right)=\left\{\left\langle x_{0}, x_{1}\right\rangle: \mathfrak{F} \vDash \Psi\left[\begin{array}{lllll}
v_{0} & v_{1} & \mathbf{v}_{0} & \ldots & \mathbf{v}_{n} \\
x_{0} & x_{1} & v_{0} & \ldots & v_{n}
\end{array}\right]\right\} \text {. }
$$

The formulas $\Phi$ and $\Psi$ are said to uniformly define a class $K_{\tilde{\mho}}^{\Phi}$ of subsets of $F \times F$ if the following condition holds: $S \in K_{\mathscr{F}}^{\Phi \Psi}$ if and only if there are elements $v_{n}, \ldots, v_{n}$ of $F$ such that

$$
\mathfrak{F} \vDash \Phi\left[\begin{array}{ccc}
\mathbf{v}_{0} & \ldots & \mathbf{v}_{n} \\
v_{0} & \ldots & v_{n}
\end{array}\right] \text { and } \quad S=S_{\mathfrak{F}}^{\Psi}\left(v_{0}, \ldots, v_{n}\right)
$$

We can express this condition equivalently in the following way: $S \in K_{\Im}^{\Phi \Psi}$ if and only if the structure $\langle F ; 0,1,+, \cdot, \leqslant, S\rangle$ is a model of the sentence

$$
\boldsymbol{K}^{\Phi \Psi}=\exists \mathbf{v}_{0} \ldots \exists \mathbf{v}_{n}\left\{\Phi \wedge \forall v_{0} \forall v_{1}\left[S\left(v_{0} v_{1}\right) \leftrightarrow \Psi\right]\right\} .
$$

The sentence $K^{\Phi \Psi}$ obviously belongs to $\mathbf{L}(\mathbf{0}, \mathbf{1},+, \cdot, \leqslant, S)$. If there are two formulas $\Phi, \Psi$ which define a class $K$ uniformly, we say that $K$ is uniformly definable in the elementary theory of fields. The class of all restricted affine planes $\mathfrak{N}_{2}(\mathfrak{F}, S)$ with $S \in K_{\mathscr{F}}^{\Phi \Psi}$ will be denoted by $\mathscr{K}_{\mathscr{F}}^{\Phi \Psi}$. Throughout this section the formulas $\Phi, \Psi$, and the sequence $\mathbf{v}_{0}, \ldots, \mathbf{v}_{n}$ in 5.6 will be regarded as fixed. The same applies to $\left\langle\mathbf{v}_{0}^{\prime}, \mathbf{v}_{1}^{\prime} \ldots\right\rangle$ wich is assumed to be the (infinite) sequence of all distinct variables not occurring free either in $\Phi$ or in $\exists v_{0} \exists v_{1} \Psi$.

Definition 5.8. Consider a unique function $\mathbf{G}$ mapping $\mathbf{L}(\boldsymbol{B})$ into $\mathbf{L}(\mathbf{0}, \mathbf{1},+, \cdot, \leqslant)$ and satisfying the following condition:

(i) $\mathbf{G}\left(B\left(v_{i} v_{j} v_{k}\right)\right)=\exists \mathbf{v}_{0}^{\prime}\left[0 \leqslant \mathbf{v}_{0}^{\prime} \wedge \mathbf{v}_{0}^{\prime} \leqslant \mathbf{1} \wedge \mathbf{v}_{2 j+1}^{\prime}+\mathbf{v}_{2 i+1}^{\prime} \cdot \mathbf{v}_{0}^{\prime}=\mathbf{v}_{2 i+1}^{\prime}+\right.$ $\left.+\mathbf{v}_{2 k+1}^{\prime} \cdot \mathbf{v}_{0}^{\prime} \wedge \mathbf{v}_{2 j+2}^{\prime}+\mathbf{v}_{2 i+2}^{\prime} \cdot \mathbf{v}_{0}^{\prime}=\mathbf{v}_{2 i+2}^{\prime}+\mathbf{v}_{2 k+2}^{\prime} \cdot \mathbf{v}_{0}^{\prime}\right]$

(ii) $\mathbf{G}(\neg \Gamma)=\neg \mathbf{G}(\Gamma)$ for any $\Gamma \in \mathbf{L}(\boldsymbol{B})$,

(iii) $\mathbf{G}(\Gamma \vee \Delta)=\mathbf{G}(\Gamma) \vee \mathbf{G}(\Delta)$ for any $\Gamma, \Delta \in \mathbf{L}(B)$,

(iv) $\mathbf{G}\left(\exists \boldsymbol{v}_{i} \Gamma\right)=\exists \mathbf{v}_{2 i+1}^{\prime} \exists \mathbf{v}_{2 i+2}^{\prime}\left[\Psi\left(\begin{array}{ll}\boldsymbol{v}_{0} & \boldsymbol{v}_{1} \\ \mathbf{v}_{2 i+0}^{\prime} & \mathbf{v}_{2 i+1}^{\prime}\end{array}\right) \wedge \mathbf{G}(\Gamma)\right]$ for any $\Gamma \in \mathbf{L}(B)$.

The following lemma is proved by induction:

LEMMA 5.9. Let $\Gamma \in \mathbf{L}(\boldsymbol{B})$ and $v_{0}, \ldots, v_{n}$ be any sequence of elements of $\mathbb{F}$. Then for any $\left\langle x_{0}, y_{0}\right\rangle, \ldots,\left\langle x_{m}, y_{m}\right\rangle$ in $S_{\widetilde{f}}^{\prime \prime}\left(v_{0}, \ldots, v_{n}\right)$ we have

$$
\mathfrak{N}\left(\widetilde{F}, S_{\mathfrak{F}}^{\Psi}\left(v_{0}, \ldots, v_{n}\right)\right) \vDash \Gamma\left[\begin{array}{lll}
v_{0} & \ldots & v_{m} \\
\left\langle x_{0}, y_{0}\right\rangle & \ldots & \left\langle x_{m}, y_{m}\right\rangle
\end{array}\right]
$$


if and only if

$$
\widetilde{F} \vDash \mathbf{G}(\Gamma)\left[\begin{array}{llllllll}
\mathbf{v}_{0} & \ldots & \mathbf{v}_{n} & \mathbf{v}_{1}^{\prime} & \mathbf{v}_{2}^{\prime} & \ldots & \mathbf{v}_{2 m+1}^{\prime} & \mathbf{v}_{2 m+2}^{\prime} \\
v_{0} & \ldots & v_{n} & x_{0} & y_{0} & \ldots & x_{m} & y_{m}
\end{array}\right]
$$

Thus, for any sentence $\Delta \in \mathbf{L}(\boldsymbol{B})$, we have

if and only if

$$
\mathfrak{A}\left(\mathfrak{\mho}, S_{\widetilde{\mho}}^{\Psi}\left(v_{0}, \ldots, v_{n}\right)\right) \vDash \Delta
$$

$$
\mathfrak{F} \vDash \mathbf{G}(\Delta)\left[\begin{array}{lll}
\mathbf{v}_{0} & \cdots & \mathbf{v}_{i n} \\
v_{0} & \ldots & v_{n}
\end{array}\right]
$$

and hence

LEMMA 5.10. For any sentence $\Delta \in \mathbf{L}(\boldsymbol{B}), \Delta \in \mathbf{T}\left(\mathscr{K}_{\sharp}^{\phi \Psi}\right)$ if and only if

$$
\forall \mathbf{v}_{0} \ldots \forall \mathbf{v}_{n}[\Phi \rightarrow \mathbf{G}(\Delta)] \in \mathbf{T}(\mathfrak{F}) .
$$

Since any two real-closed fields are elementarily equivalent, we get, as a simple consequence of Lemma 5.10,

COROLLARY 5.11. For any two real-closed fields $\mathfrak{F}$ and $\mathfrak{F}^{\prime}$ and any two formulas $\Phi, \Psi \in \mathbf{L}(\mathbf{0}, \mathbf{1},+, \cdot, \leqslant)$, we have $\mathbf{T}\left(\mathscr{K}_{\Im}^{\Phi \Psi}\right)=\mathbf{T}\left(\mathscr{K}_{\mho^{\prime}}^{\Phi \Psi^{\prime}}\right)$.

THEOREM 5.12. Let $\mathfrak{F}$ be any real-closed field, and let every element of $K_{\mathfrak{F}}^{\Phi \Psi}$. be a convex set in $\mathfrak{I}_{2}(\mathfrak{F})$. Then the theory $\mathbf{T}\left(\mathscr{K}_{\mathscr{F}}^{\Phi \Psi}\right)$ satisfies the following conditions:

(i) $\mathbf{T}\left(\mathscr{K}_{\mathbb{\xi}}^{\Phi \Psi}\right)$ is a finite extension of $\mathbf{G A}_{2}$,

(ii) $\mathbf{T}\left(\mathscr{K}_{\tilde{\xi}}^{\Phi \Psi}\right)$ is not finitely axiomatizable,

(iii) $\mathbf{T}\left(\mathscr{K}_{\widetilde{\mho}}^{\Phi \Psi}\right)$ is decidable.

(iv) if, for any $S^{\prime}, S^{\prime \prime} \in K_{\mathscr{F}}^{\Phi \Psi}$ the corresponding restricted affine spaces $\mathfrak{Q}_{2}\left(\mathfrak{F}, S^{\prime}\right)$ and $\mathfrak{U}_{2}\left(\mathfrak{F}, S^{\prime \prime}\right)$ are isomorphic (or at least elementarily equivalent), then $\mathbf{T}\left(\mathscr{K}_{\Im}^{\Phi \Psi}\right)$ is complete.

Proof. (i) Consider $K_{\mathfrak{R}}^{\Phi \Psi}$ where $\Re$ is the field of real numbers. Since $\mathfrak{F}$ is a real closed field (and therefore elementarily equivalent to $\mathfrak{R}$ ) and every element of $K_{\mathfrak{J}}^{\Phi \Psi}$ is a convex set in $\mathfrak{I}_{2}(\mathscr{\Im})$, then, by Corollary 5.11 , it follows that any element of $K_{\mathscr{P}}^{(\Phi)}$ is a convex set in $\mathfrak{Q}_{2}(\mathfrak{R})$. By Theorem 3.10 (i) all relational systems from the class $\mathscr{K}_{\mathfrak{Q}}^{\Phi \Psi}$ are models of $\mathbf{G A}_{2}$, and so $\mathbf{T}\left(\mathscr{K}_{\Re}^{\Phi \Psi}\right)$ is an extension of $\mathbf{G A}_{2}$. By Corollary 5.11 $\mathbf{T}\left(\mathscr{K}_{\mathscr{R}^{\Phi}}^{\Phi \Psi}\right)=\mathbf{T}\left(\mathscr{K}_{\mathscr{F}}^{\Phi \Psi}\right)$, and therefore $\mathbf{T}\left(\mathscr{K}_{\tilde{F}}^{\Phi \Psi}\right)$ is an extension of $\mathbf{G A}_{2}$. To prove that

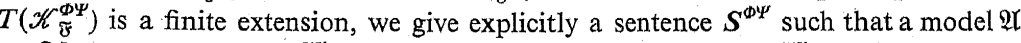
of $\mathbf{G A}_{2}$ is a model of $S^{\Phi \Psi}$ if and only if it is a model of $\mathbf{T}\left(\mathscr{K}_{\mathscr{F}}^{\Phi \Psi}\right)$ :

$$
\left.S^{\Phi \Psi}=\right\urcorner \llbracket \neg\left\{\mathbf{H F}(\Phi) \wedge \forall v_{8} \forall v_{9} \forall v_{10} \forall v_{11}\left[\left(\exists v_{12} K\right) \leftrightarrow \mathbf{H F}(\Psi)\right]\right\} \rrbracket
$$

(see Lemmas 2.18 and 5.6).

(ii) Suppose that $\mathbf{T}\left(\mathscr{K}_{\mathscr{F}}^{\Phi \Psi}\right)$ is finitely axiomatizable (i.e. there is a sentence $\Delta$ such that $\mathfrak{N} \vDash \Delta$ if and only if $\mathfrak{N}$ is a model of $\mathrm{T}\left(\mathscr{K}_{\mathbb{F}}^{\Phi \Psi}\right)$. By Lemma 5.10 and Corollary 5.11, $\mathfrak{F} \vDash \llbracket \Phi \rightarrow \mathbf{G}(\Delta) \rrbracket$ if and only if $\mathfrak{F}$ is a real-closed field. Therefore the theory of real-closed fields would be finitely axiomatizable, which contradicts a wellknown result (cf. Tarski [16] where a proof of the related Theorem 4 is given). (iii) By Lemma 5.10 a $\Delta \in \mathbf{T}\left(\mathscr{K}_{\mathscr{F}}^{\Phi \Psi}\right)$ if and only if $\llbracket \Phi \rightarrow \mathbf{G}(\Delta) \rrbracket \in \mathbf{T}(\mathscr{F})$. Since $\mathfrak{F}$ is a real closed field, its theory is decidable (see Tarski [15]); therefore $\mathbf{T}\left(\mathscr{K}_{\widetilde{\Psi}}^{\Phi \Psi}\right)$ is decidable.

(iv) Obvious.

The theory $\mathbf{T}\left(\mathscr{K}_{\mathscr{F}}^{\Phi \Psi}\right)$ can be called the (elementary) affine geometry of points sets in $K_{\tilde{F}}^{\Phi \Psi}$.

It may be noticed that the notion of uniform definability could be referred not to a class of subsets $S$ of $F \times F$ but to a class of restricted spaces $\mathfrak{A}_{2}(\mathfrak{F}, S)$ with $\mathfrak{F}$ now ranging over a class of real-closed ordered fields (possibly over the class of all such fields). In this way we could obtain a seemingly more general result, which, however, would not essentially improve Theorem 5.12 (cf. Corollary 5.11).

§ 6. Examples of extending $\mathbf{G A}_{2}$. By Theorem 5.12(i), the theory. $\mathbf{T}\left(\mathscr{K}_{\tilde{\delta}}^{\Phi \Psi}\right)$ is axiomatizable, and an axiom system for this theory can be obtained by adjoining finitely many sentences or, what amounts to the same, a single sentence $\chi\left(\mathscr{K}_{\mathscr{F}}^{\Phi \Psi}\right)$ to the axiom system of $\mathbf{G A}_{2}$. The proof of Theorem 5.12 gives an effective construction of such a sentence dependent on the defining formulas $\Phi$ and $\Psi$; frequently, however, a simpler sentence serving the same purpose can be obtained by some other method.

We shall give below a few simple and interesting examples of classes $\mathscr{K}$ satisfying the hypothesis of Theorem 5.12, and in each case we shall formulate an appropriate sentence $\dot{\chi}(\mathscr{K})$. For convenience we may take $\mathfrak{R}$ for $\mathscr{F}$, and identify the corresponding classes $\mathscr{K}_{\Im}^{\Phi \Psi}$ and $\mathscr{K}_{\Re}^{\Phi \Psi}$.

EXAMPLE 6.1. $\mathscr{K}_{1}$ contains the whole plane as the only member. The theory $\mathbf{T}\left(\mathscr{K}_{1}\right)$, the affine geometry of the plane, clearly coincides with $\mathbf{A E}_{2}$; as we know, for $\chi\left(\mathscr{K}_{1}\right)$ we can take Euclid's axiom in the form

$E=\forall t x y z u \exists v w[B(x u t) \wedge B(y u z) \wedge \neg x=u \rightarrow B(x y v) \wedge B(x z w) \wedge B(v t w)]$.

Obviously $\mathscr{K}_{1}$ satisfies the premiss of Theorem 5.12 (iv) and therefore $\mathbf{T}\left(\mathscr{K}_{1}\right)$ is complete.

EXAMPLE 6.2. $\mathscr{K}_{2}$ consists of all interiors of halfplanes. For $\chi\left(\mathscr{K}_{2}\right)$ we can take the conjunction of the sentence

$\exists x y \forall z u v w \exists t[\neg x=y \wedge\{B(x z v) \wedge B(u v w) \wedge \neg z=v \rightarrow[B(u z t) \vee B(w z t)] \wedge$

$\wedge[B(x y t) \vee B(y t x) \vee B(t x y)]\}]$

and the negation of Euclid's axiom. Again $\mathbf{T}\left(\mathscr{K}_{2}\right)$ is complete.

EXAMPLE 6.3. $\mathscr{K}_{3}$ consists of all interiors of circles. Clearly $\mathbf{T}\left(\mathscr{K}_{3}\right)$ coincides. with the affine hyperbolic geometry $\mathbf{A H}_{2}$. It can be shown that for $\chi\left(\mathscr{K}_{3}\right)$ we can take the conjunction of the following two sentences:

$$
\begin{aligned}
H_{1}=\forall x y & \exists z u v w \\
& \forall t[\neg x=y \rightarrow B(x z v) \wedge B(u v w) \wedge \neg z=u \wedge \\
& \wedge\{[\neg B(u z t) \wedge \neg B(w z t)] \vee[\neg B(x y t) \wedge \neg B(y t x) \wedge \neg B(t x y)]\}],
\end{aligned}
$$$$
H_{2}=\forall x y z t u v w[t x \text { 年 } y v \wedge v x \uparrow y t \wedge u z \uparrow y w \wedge w z \Uparrow y u \wedge x v \Uparrow z w \wedge x t \uparrow z u \wedge
$$

$\wedge B(t y w) \wedge B(v y u) \rightarrow B(x y z)]$ 
Notice that $\boldsymbol{H}_{1}$ is the negation of the sentence formulated above in Example 6.2; on the basis of $\mathbf{G A}_{2}$ it is stronger than the negation of Euclid's axiom. On the other hand, $\boldsymbol{H}_{2}$ is a special form of Pascal's theorem involving six paits of parallel halflines.

It is important to observe that, from a mathematical viewpoint, $\mathbf{H}_{2}$ and $\mathbf{A} \mathbf{H}_{2}$ are essentially the same theories since it is known that $\boldsymbol{D}$ is definable in $\mathbf{H}_{2}$ in terms of $\boldsymbol{B}$ (see Menger [5] and Royden [8]). Hence the axiom system consisting of $\mathbf{A}_{1}-\mathbf{A}_{8}, \boldsymbol{H}_{1}, \boldsymbol{H}_{2}$ and the (non-elementary) continuity axiom can be regarded as an adequate axiom system for the ordinary (non-elementary) two-dimensional hyperbolic geometry.

Another consequence of this observation is that the metamathematical properties of $\mathbf{A H}_{2}$ resulting from Theorem 5.12 can also be derived directly from the corresponding theorems on $\boldsymbol{H}_{2}$ stated in Szmielew [14]

EXAMPLE 6.4. $\mathscr{K}_{4}$ is the class consisting of the plane and of all interiors of halfplanes and circles. As $\chi\left(\mathscr{K}_{4}\right)$ we can take Pascal's theorem in the special form stated above in Example 6.3. On the other hand, let $\mathscr{K}_{4}^{\prime}$ be the class consisting of the plane 'and of all interiors of circles; then $\mathbf{T}\left(\mathscr{K}_{4}^{\prime}\right)$ clearly coincides with $\mathbf{A A}_{2}$ and for $\chi\left(\mathscr{K}_{4}^{\prime}\right)$ we can take the conjunction of Pascal's theorem and the negation of $\chi\left(\mathscr{K}_{2}\right)$. It might be interesting to find a simpler sentence for this purpose. Obviously neither $\mathbf{T}\left(\mathscr{K}_{4}\right)$ nor $\mathrm{T}\left(\mathscr{K}_{4}^{\prime}\right)$ is complete; $\mathbf{T}\left(\mathscr{K}_{4}\right)$ has three complete and consistent extensions, $\mathbf{T}\left(\mathscr{K}_{1}\right), \mathbf{T}\left(\mathscr{K}_{2}\right)$ and $\mathbf{T}\left(\mathscr{K}_{3}\right)$, while $\mathbf{T}\left(\mathscr{K}_{4}^{\prime}\right)$ has two such extensions, $\mathbf{T}\left(\mathscr{K}_{1}\right)$ and $\mathbf{T}\left(\mathscr{K}_{3}\right)$.

EXAMPLE 6.5. Let $n \geqslant 3$, let further $\mathscr{P}_{n}$ be the class of all interiors of convex polygons with at most $n$ vertices, and $\mathscr{P}_{n}^{\prime}$ the class of all interiors of convex polygons with exactly $n$ vertices. For $\chi\left(\mathscr{P}_{n}\right)$ we can take

$$
\exists v_{0} \ldots v_{n} \forall v_{n+1} v_{n+2} \exists v_{n+3}\left\{\neg v_{0}=v_{1} \wedge \ldots \wedge \neg v_{0}=v_{n} \wedge\left[B\left(v_{1} v_{0} v_{n+3}\right) \vee \ldots\right.\right.
$$

$\left.\left.\ldots \vee B\left(v_{n} v_{0} v_{n+3}\right)\right] \wedge\left[B\left(v_{n+1} v_{n+2} v_{n+3}\right) \vee B\left(v_{n+2} v_{n+3} v_{n+1}\right) \vee B\left(v_{n+3} v_{n+1} v_{n+2}\right)\right]\right\}:$

For $n=3$ the class $\mathscr{P}_{n}$ coincides with $\mathscr{P}_{n}^{\prime}$; if $n>3$, we take for $\chi\left(\mathscr{P}_{n}^{\prime}\right)$ the conjunction of $\chi\left(\mathscr{P}_{n}\right)$ and the negation of $\chi\left(\mathscr{P}_{n-1}\right)$. The classes $\mathscr{P}_{3}^{\prime}$ and $\mathscr{P}_{4}^{\prime}$ satisfy the premiss of Theorem 5.12(iv), so the corresponding theories $\mathbf{T}\left(\mathscr{P}_{3}^{\prime}\right)$ and $\mathbf{T}\left(\mathscr{P}_{4}^{\prime}\right)$, the affine geometries of triangles and quadrangles, are complete. On the other hand, all the theories $\mathbf{T}\left(\mathscr{P}_{n}^{\prime}\right)$ with $n \geqslant 5$ are incomplete and each of them has $2^{N_{0}}$ complete and consistent extensions (compare the proof of Theorem 4.10).

It may be mentioned that none of the classes discussed in Examples 3-5 satisfies the following condition: together with any set $S$ it should contain all the sets $X$ such that the space $\mathfrak{I}_{2}(\mathfrak{R}, S)$ and $\mathfrak{I}_{2}(\mathfrak{R}, X)$ are isomorphic. Hence each of the classes $\mathscr{K}$ can be enlarged by some subsets of $R \times R$ without affecting the corresponding theory $\mathbf{T}(\mathscr{K})$

There are interesting extensions of $\mathbf{G A}_{2}$ which are not of the form discussed in Theorem 5.12 but are obtained by direct enlargement of the axiom system. Again we wish to give a few examples.
EXAMPLE 6.6. In the axiom system of $\mathbf{G A}_{2}$ we include the negation of Euclid's axiom. Let $\mathbf{T}_{(1)}$ be the resulting theory. Every model of $\mathbf{T}_{(1)}$ is isomorphic to a restricted affine space $\mathfrak{I}_{2}(\tilde{F}, S)$ where $S$ is different from the whole plane $F \times F$ (and, of course, $\mathfrak{F}$ is a real closed field). The axiom system for $\mathbf{T}_{(1)}$ has been constructed by the same method as the axiom system for $\mathbf{H}_{2}$ in Szmielew [14]; nevertheless, the two theories differ essentially in some of their metamathematical properties. Using the methods employed in the proofs of Theorems $4.10,4.15$ and 4.16 , we may prove that $\mathbf{T}_{(1)}$ is hereditarily undecidable, non-finitely axiomatizable, and has $2^{\text {s。 }}$ complete extensions.

In describing the remaining three examples we shall use $\boldsymbol{E}$ as an abbreviation of the formula

$$
\forall t u \exists v w[B(x u t) \wedge B(y u z) \wedge \neg x=u \rightarrow B(x y v) \wedge B(x z w) \wedge B(v t w)] .
$$

As is easily seen, three points $x, y, z$ of an affine space (i.e. a model of $\mathbf{G A}_{2}$ ), in case they are not collinear, satisfy this formula if and only if the angle whose vertex is $x$ and whose sides pass, respectively, through $y$ and $z$, is a "Euclidean angle" in the sense that every point $t$ inside this angle lies between some points $v$ and $w$ lying on the two sides of this angle. Using the abbreviation $\boldsymbol{E}$ we can give to Euclid's axiom $\boldsymbol{E}$ the following simple form:

$$
\forall x y z E(x y z) \text {. }
$$

EXAMPLE 6.7. We supplement the axiom system of $\mathbf{G A}_{2}$ by the sentence

$$
\forall x r s \exists y z[\neg r=s \wedge \neg B(x r s) \rightarrow B(r y s) \wedge B(r z s) \wedge \neg y=z \wedge E(x y z)] .
$$

Let $\mathbf{T}_{(2)}$ be the theory based upon the extended axiom system. Using Theorem 3.10, we easily show that every model of $\mathbf{T}_{(2)}$ is isomorphic to a restricted affine space $\mathfrak{I}_{2}(\mathfrak{F}, S)$ in which the boundary of $S$ is rectilinear, i.e., is the closure of a union of segments with distinct endpoints; conversely, every restricted affine space (over a real-closed ordered field) with this property is a model of $\mathbf{T}_{(2)}$ provided it is a model of $\mathbf{G A}_{2}$. Again using the methods employed in the proofs of Theorems $4.10,4.15$ and 4.16 , we may prove that the theory $\mathbf{T}_{(2)}$ is hereditarily undecidable, non-finitely axiomatizable, and has $2^{N_{0}}$ complete and consistent extensions.

EXAMPLE 6.8. We now enrich the axiom system of $\mathbf{G A}_{2}$ by including the sentence

$$
\forall x y s \exists z[\neg y=s \wedge \neg B(x y s) \rightarrow B(y z s) \wedge \neg y=z \wedge E(x y z)]
$$

The resulting theory $\mathbf{T}_{(3)}$, which is a subtheory of $\mathbf{T}_{(2)}$ exhibits some interesting model-theoretical properties. A necessary and sufficient condition for a restricted affine space $\mathfrak{R}_{2}(\mathfrak{R}, S)$ to be a model of $\mathbf{T}_{(3)}$ is that $\mathfrak{R}_{2}(\mathfrak{R}, S)$ be isomorphic to a restricted affine space $\mathfrak{U}_{2}\left(\mathfrak{R}, S^{\prime}\right)$ where $S^{\prime}$ is the interior of a convex polygon, i.e., $S^{\prime} \in P_{n}$ for some $n>3$ (see Example 6.5). However, there is a model of $\mathbf{T}_{(3)}$ in which all the sentences $\chi\left(\mathscr{P}_{n}\right)$ for $n=3,4, \ldots$ are false. Indeed, let us enrich the theory $\mathbf{T}_{(3)}$ by including the negations of all the sentences $\chi\left(\mathscr{P}_{n}\right), n=3,4, \ldots$ Since any finite set of sentences $\chi\left(\mathscr{P}_{n}\right)$ is consistent with $\mathbf{T}_{(3)}$, the resulting theory $\mathbf{T}_{(4)}$ is consistent 3 - Fundamenta Mathematicae CV/3 
but has no models in the class $\mathscr{L}$ of all restricted affine spaces over $\mathfrak{R} . \mathbf{T}_{(4)}$ is no a finite extension of $\mathbf{G A}_{2}$. Using the method employed in the proof of Theorem 5.10, we may prove that $\mathbf{T}_{(3)}$ has $2^{N_{0}}$ complete and consistent extensions, and by a method similar to that employed in the proof of Theorem 5.12, and we may conclude that both $\mathbf{T}_{(3)}$ and $\mathbf{T}_{(4)}$ are not finitely axiomatizable. The problem of decidability remains open for both theories.

EXAMPLE 6.9. Finally consider the theory $\mathbf{T}_{(5)}$ obtained by adding to the axioms of $\mathbf{G A}_{2}$ the sentence

$$
\forall x y z[E(x y z) \rightarrow B(x y z) \wedge B(y z x)] .
$$

Every model of $\mathbf{T}_{(5)}$ is isomorphic to a restricted affine space $\mathfrak{O}_{2}(\mathfrak{F}, S)$ in which (i) the boundary of $S$ is curvilinear, i.e., does not include any segment with distinct endpoints, and (ii) $S$ does not include any two distinct halflines of the space $\mathfrak{S}_{2}(\mathfrak{F})$ which have the same origin and do not lie on the same line. Conversely, every restricted affine space (over a real-closed ordered field) which has this properties and is a model of $\mathbf{G A}_{2}$ is also a model of $\mathbf{T}_{(5)}$. The theory $\mathbf{T}_{(5)}$ is hereditarily undecidable, non-finitely axiomatizable, and has $2^{\mathrm{N}_{0}}$ complete and consistent extensions. The proof of non-finite axiomatizability does not differ essentially from that of Theorem 5.14. To prove that $\mathbf{T}_{(5)}$ is hereditarily. undecidable and has $2^{\aleph_{0}}$ complete extensions we must first modify the proofs of Theorems 4.10 and 4.15 by taking models whose boundaries are sums of circle segments instead of line segments. Then we employ the technique developed at the beginning of Section 5 to define a counterpart of the notion of vertex.

To conclude we state three results of a different character concerning extensions of $\mathbf{G A}_{2}$. Let $\mathfrak{F}=\langle F, 0,1,+, \cdot, \leqslant\rangle$ be a real closed field; further, let

$$
1 \quad K_{\widetilde{\Psi}}^{r}=\left\{\left\langle x_{1}, x_{2}\right\rangle: x_{1}, x_{2} \in F \wedge x_{1}^{2}+x_{2}^{2}<r^{2}\right\} .
$$

LEMMA 6.10. For any $r \in F$, the systems $\mathfrak{I}_{2}(\mathfrak{F})$ and $\mathfrak{N}_{2}\left(\mathfrak{F}, K_{\mathfrak{F}}^{r}\right)$ are elementarily equivalent with respect to universal sentences.

Proof. Let $\mathfrak{F}^{\prime}$ be a real-closed field, which is an extension of $\mathfrak{F}$ and has an element $r^{\prime}$ greater than all elements of $\mathfrak{F}$. Then

$$
\mathfrak{N}_{2}\left(\mathfrak{F}, K_{\widetilde{F}}^{r}\right) \subseteq \mathfrak{N}_{2}(\mathfrak{F}) \subseteq \mathfrak{N}_{2}\left(\mathfrak{F}^{\prime}, K_{\mathfrak{F}^{\prime}}^{r^{\prime}}\right) \text {. }
$$

Both $\mathfrak{I}_{2}\left(\widetilde{F}, K_{\mathscr{\mho}}^{r}\right)$ and $\mathfrak{V}_{2}\left(\mathscr{F}^{\prime}, K_{\mathfrak{F}^{\prime}}^{r^{\prime}}\right)$ are models of the theory $\mathbf{T}\left(\mathscr{K}_{3}\right)$ discussed in Example 6.3. Since the theory $\mathbf{T}\left(\mathscr{K}_{3}\right)$ is complete, $\mathfrak{N}_{2}\left(\mathfrak{F}, K_{\mathfrak{F}}^{r}\right)$ and $\mathfrak{A}_{2}\left(\mathfrak{F}^{\prime}, K_{\mathfrak{F}^{\prime}}^{r^{\prime}}\right)$ are elementarily equivalent. Therefore $\mathfrak{N}_{2}\left(\mathfrak{F}, K_{\mathfrak{F}}^{r}\right)$ and $\mathfrak{Q}_{2}(\mathfrak{F})$ are elementarily equivalent with respect to universal sentences.

THEOREM 6.11. If a universal sentence is true in some model of $\mathbf{G A}_{2}$, then it is true in every model of $\mathbf{G A}_{2}$. In other words, $\mathbf{G A}_{2}$ is complete with respect to universal sentences.

A related result was stated in Tarski [16] (Theorem 7).

Proof. Let $\mathfrak{Q}$ be a model of $\mathbf{G A}_{2}$. Then by Theorem 3.9 there is a field $\mathfrak{F}$ and a set $S$ convex in $\mathfrak{I}_{2}(\mathfrak{F})$ such that $\mathfrak{Y} \cong \mathfrak{A}_{2}(\mathfrak{F}, S)$. Since $S$ is a non-empty open set, there is an element $r$ of the field $\mathfrak{F}$, such that $\mathfrak{A}_{2}\left(\mathfrak{F}, K_{\mathfrak{F}}^{r}\right)$ may be embedded in $\mathfrak{N}_{2}(\mathfrak{F}, S) \subseteq \mathfrak{U}_{2}(\mathfrak{F})$. Thus, by Lemma $6.10, \mathfrak{N}_{2}(\mathfrak{F}, S)$, i.e. $\mathfrak{A}$ itself, is elementarily equivalent to $\mathfrak{A}_{2}(\mathscr{F})$ with respect to universal sentences. Since for any two real-closed fields $\mathfrak{F}$ and $\mathfrak{F}^{\prime}$, the relational systems $\mathfrak{H}_{2}(\mathfrak{F})$ and $\mathfrak{A}_{2}\left(\mathfrak{F}^{\prime}\right)$ are elementarily equivalent, it follows that any two models of $\mathbf{G A}_{2}$ are elementarily equivalent with respect to universal sentences.

THEOREM 6.12. Let $\Psi$ be a sentence of the form

$$
\forall \mathbf{v}_{1} \ldots \forall \mathbf{v}_{k} \exists \mathbf{v}_{k+1} \ldots \exists \mathbf{v}_{k+n} \Phi,
$$

where $\Phi$ is a formula without quantifiers, and let $m$ be any integer $\geqslant k$ and $\geqslant 3$. Then the sentence $\Psi$ is valid in $\mathbf{G A}_{2}$ if and only if it is valid in the theory $\mathbf{T}\left(\mathscr{P}_{m}\right)$ of Example 6.8.

Proof. Since $\mathbf{T}\left(\mathscr{P}_{m}\right)$ is an extension of $\mathbf{G A}_{2}$, it will suffice to prove that if $\Psi$ is not a theorem of $\mathbf{G A}_{2}$ then it is not a theorem of $\mathbf{T}\left(\mathscr{P}_{m}\right)$. Let $\mathfrak{A}$ be a model of $\mathbf{G A}_{2}$ such that $\Psi$ is false in $\mathfrak{N}$. By Theorem 3.9 we may assume that $\mathfrak{A}=\mathfrak{A}_{2}(\mathfrak{F}, S)$ for some real-closed field $\mathfrak{F}$, and a point set $S$ convex in $\mathfrak{A}_{2}(\mathfrak{F})$. Since the sentence is not true in $\mathfrak{N}$, we have $\left.\mathfrak{Q} \vDash \exists v_{1} \ldots \exists v_{k} \forall v_{k+1} \ldots \forall v_{k+n}\right\urcorner \Phi$. Assuming, without loss of generality, that $\mathbf{v}_{1}, \ldots, \mathbf{v}_{k}$ are pair-wise distinct, this means that there are points $x_{1}, \ldots, x_{k} \in S$ such that

$$
\left.\mathfrak{N} \vDash \forall \boldsymbol{v}_{k+1} \ldots \forall v_{k+n}\right\urcorner \Phi\left[\begin{array}{lll}
\mathbf{v}_{1} & \ldots & \mathbf{v}_{k} \\
x_{1} & \ldots & x_{k}
\end{array}\right] .
$$

The set $S$ is open and convex, and so it includes a set $P$, the interior of a convex polygon with $m$ vertices, containing all the points $x_{1}, \ldots, x_{k}$. Since $\mathfrak{H}_{2}(\widetilde{\mho}, P) \subseteq \mathfrak{H}_{2}(\mathfrak{F}, S)$, we have

$$
\mathfrak{V}_{2}(\widetilde{J}, P) \vDash \forall v_{k+1} \ldots \forall v_{k+n} \neg \Phi\left[\begin{array}{lll}
\mathbf{v}_{1} & \ldots & \mathbf{v}_{k} \\
x_{1} & \ldots & x_{k}
\end{array}\right],
$$

and therefore the sentence $\Psi$ is false in $\mathfrak{H}_{2}(\mathscr{F}, P)$. Thus $\Psi$ is not a theorem of $\mathbf{T}\left(\mathscr{P}_{m}\right)$.

Now $\mathbf{T}\left(\mathscr{P}_{m}\right)$ is decidable (see Theorem 5.12(iii)), and so Theorem 6.12 implies

THEOREM 6.13. The theory $\mathbf{G A}_{2}$ is decidable with respect to the set of $\forall \exists$-sentences (in other words, the set of $\forall \exists$-sentences which are valid in $\mathbf{G A}_{2}$ is recursive).

THEOREM 6.14. The theory $\mathbf{G A}_{2}$ is not a $\forall \exists$-theory.

Proof. Let $\mathbb{F}=\langle F, 0,1,+, \cdot, \leqslant\rangle$ be a field of algebraic numbers, and. let $\alpha$ be any transcendental number. There is an increasing sequence $\alpha_{i}$ of rational numbers converging to $\alpha$. Let

$\dot{S}_{i}=\left\{\left\langle x_{1}, x_{2}\right\rangle: x_{1}, x_{2} \in F \wedge x_{1}<\alpha_{i}\right\} \quad$ and $S=\left\{\left\langle x_{1}, x_{2}\right\rangle: x_{1}, x_{2} \in F \wedge x_{1}<\alpha\right\}$.

For any natural number $i, \mathfrak{A}_{i}=\mathfrak{Q}_{2}\left(\mathfrak{F}, S_{i}\right)$ is a model of $\mathbf{G A}_{2}$ (see Example 6.5); on the other hand, $U \mathfrak{A A}_{i}=\mathfrak{2}_{2}\left(\mathfrak{F}, S\right.$ ) is not a model of $\mathbf{G A}_{2}$ (cf. the proof of Theorem 3.10(ii)). Thus by the Łoś-Suszko Theorem (see Łoś-Suszko [4]) $\mathbf{G A}_{2}$ is not a $\forall \exists$-theory. 
§ 7. Multi-dimensional geometries $\mathbf{G A}_{n}$. In this scction we shall indicate how to extend some of our results to the case of $n$-dimensional affine geometry for $n \geqslant 3$.

Defintarion 7.1. To define conveniently the notion of $n$-dimensional general affine geometry we introduce a sequence of abbreviations (cf. Kordos [3]):

$$
\begin{aligned}
& L_{1}=B\left(\mathbf{v}_{1} \mathbf{v}_{2} \mathbf{v}_{3}\right) \vee B\left(\mathbf{v}_{2} \mathbf{v}_{3} \mathbf{v}_{1}\right) \vee B\left(\mathbf{v}_{3} \mathbf{v}_{1} \mathbf{v}_{2}\right), \\
& L_{n}=\exists \mathbf{v}_{0} \bigvee_{\left\langle i_{1}, i_{n+2}\right\rangle}\left[L_{n-1}\left(\mathbf{v}_{0} \mathbf{v}_{i_{1}} \ldots \mathbf{v}_{i_{n}}\right) \wedge L_{1}\left(\mathbf{v}_{0} \mathbf{v}_{i_{n+1}} \mathbf{v}_{i_{n+2}}\right)\right]
\end{aligned}
$$

for $n \geqslant 1$, where $\bigvee_{\left\langle i_{1} \ldots i_{n+2}\right\rangle} \Phi_{\left\langle i_{1} \ldots i_{n+2}\right\rangle}$ denotes the disjunction of all formulas $\Phi_{\left\langle i_{1} \ldots i_{n+2}\right\rangle}$ with $\left\langle i_{1} \ldots i_{n+2}\right\rangle$ a permutation of $\{1, \ldots, n+2\}$. It is easy to see that $L_{1}$ denotes the notion of collinearity, $L_{2}$ the notion of coplanarity and, in general, $L_{n}$ the notion of belonging to the same $n$-dimensional hyperplane.

Definition 7.2. By the $n$-dimensional general affine geometry $\mathbf{G A}_{\|}$we mean a theory formulated in the first order language $\mathbf{L}(\boldsymbol{B})$ and based upon the axioms $\mathbf{A}_{1}-\mathbf{A}_{9}$ with the axioms $\mathbf{A}_{7}$ and $\mathbf{A}_{8}$ replaced by

$$
\begin{aligned}
& \left.\mathbf{A}_{7}^{n}=\exists \mathbf{v}_{1} \ldots \mathbf{v}_{n+1}\right\urcorner L_{n-1}, \\
& \mathbf{A}_{8}^{n}=\forall \mathbf{v}_{1} \ldots \mathbf{v}_{n+2} L_{n} .
\end{aligned}
$$

It should be pointed out that, as is well known, Desargues' axiom $\mathbf{A}_{6}$ follows from the remaining ones in $\mathbf{G A}_{n}$ for all $n>2$, and therefore it may be dropped from the axiom systems of these theories altogether (see e.g. Whitehead [19]).

It is easy to see that $\mathbf{A}_{7}^{2}$ and $\mathbf{A}_{8}^{2}$ are equivalent to $\mathbf{A}_{7}$ and $\mathbf{A}_{8}$, respectively.

The sentences $\mathbf{A}_{8}^{n}$ may easily be expressed in a $\forall \exists$-form. Kordos [3] showed that the sentences $\mathbf{A}_{7}^{n}$ cannot be expressed in such a form. He proved that any $\forall \exists$-sentence valid in $\mathbf{A E}_{n}$ is valid in $\mathbf{A E}_{m}$ whenever $2 \leqslant m<n$ (see Kordos [3] Theorem 5), where by $\mathbf{A E}_{n}$ we mean the $n$-dimensional Euclidean affine geometry, an extension of $\mathbf{G A}_{n}$ by Euclid's axiom $\boldsymbol{E}$ (see Example 6.1).

Definition 7.3. Let $\mathfrak{A}=\langle A, B\rangle$ be a model of $\mathbf{G A}_{n}$, and suppose that $a, b, c \in A$ are non-collinear, i.e.,

$$
\mathfrak{N} \vDash \neg L_{1}\left[\begin{array}{lll}
\mathbf{v}_{1} & \mathbf{v}_{2} & \mathbf{v}_{3} \\
a & b & c
\end{array}\right] .
$$

By the plane through $a, b, c$ we mean the set of all points of the model $\mathfrak{U}$, coplanar with the points $a, b$ and $c$, i.e., the set

$$
P(a b c)=\left\{x: x \in A \text { and } \mathfrak{S} \vDash L_{2}\left[\begin{array}{llll}
\mathbf{v}_{1} & \mathbf{v}_{2} & \mathbf{v}_{3} & \mathbf{v}_{4} \\
a & b & c & x
\end{array}\right]\right\} .
$$

THEOREM 7.4. If $\mathfrak{i}$ is a model of $\mathbf{G A}_{n}$ and $a, b, c \in A$ are non-collinear, then $\mathfrak{i}$ restricted to $P(a b c)$ is a model of $\mathbf{G A}_{2}$.

Using this theorem, we can generalize Theorem 3.9 to arbitrary $n \geqslant 2$ (the notions of an $n$-dimensional affine space over the field $\mathfrak{F}$ and an $n$-dimensional $S$-restricted affine space over $\mathfrak{F}$ are assumed to be understood):
THEOREM 7.5. If $\mathfrak{Y}$ is a model of $\mathbf{G A}_{n}$, then there is an ordered, real-closed field $\mathfrak{F}$ and a point set $S$ convex in $\mathfrak{N}_{n}(\mathfrak{F})$ and such that $\mathfrak{Y}$ is isomorphic to $\mathfrak{N}_{n}(\mathfrak{F}, S)$.

Repeating (with obvious changes) the proof of Theorem 3.10, we get

THEOREM 7.6. (i) Every n-dimensional $S$-restricted affine space over $\mathfrak{R}$ is a model of $\mathbf{G A}_{n}$ provided the point set $S$ is convex in $\mathfrak{N}_{n}(\mathfrak{R})$.

(ii) If the real-closed field $\mathfrak{F}$ is not isomorphic to $\mathfrak{R}$, then there is an $n$-dimensional restricted affine space over the field $\mathfrak{F}$ which is not a model of $\mathbf{G A}_{n}$.

Similarly, modifying the proofs of other relevant theorems, we get

THEOREM 7.7. The theory $\mathbf{G A}_{n}$ is

(i) undecidable,

(ii) decidable with respect to $\forall \exists$-sentences,

(iii) incomplete and, in fact, has $2^{\text {No }}$ complete extensions,

(iv) complete with respect to universal sentences,

(v) not finitely axiomatizable,

(vi) not a $\forall \exists$-theory.

\section{References}

[1] L. Henkin, J. D. Monk and A. Tarski, Cylindric algebras, Part I, North-Holland, Amsterdam-London 1971.

[2] D. Hilbert, The foundations of geometry, The Open Court La Scalle Illinois, 1962.

[3] M. Kord os, On the syntactic form of dimension axiom for affine geometry, Bull. Acad. Polon. Sci. Sér. Sci. Math. Astronom. Phys. 18 (1969), pp. 833-837.

[4] J. Łos and R. Suszko, On the extending of models (IV), Fund. Math. 44 (1957), pp. 52-60.

[5] $\mathrm{K}$. Menger, A new foundation of non-Euclidean, affine, real projective, and Euclidean geometry, Proc. National Acad. Sci. 4 (1938), pp. 486-490.

[6] A. Robins on, Introduction to model theory and to the metamathematics of algebra, North Holland, Amsterdam 1963.

[7] A. Prestel and L.W. Szczerba, Non-axiomatizability of real general affine geometry, Fund. Math. 104 (1979), pp. 193-202.

[8] H. L. Royden, Remarks on primitive notions for elementary Euclidean geometry, The axiomatic method with special reference to geometry and physics, Proceedings of an International Symposium held at the University of California, Berkeley, December 26, 1957 - January 4, 1958, North Folland, Amsterdam 1959, pp. 86-96.

[9] L. W. Szczerba, Metamatematyczne wlasności geometrii desarguesowskiej (Metamathematical properties of Desurgues geometry, in Polish), Doctoral dissertation submitted to the Mathematical Institute of the University of Warsaw in 1968, pp. 149.

[10] - Filling of gaps in ordered fields, Bull. Acad. Polon. Sci. Sér. Sci. Math. Astronom. Phys. 18 (1970), pp. 349-352.

[11] - Weak general affine geometry, Bull. Acad. Polon. Sci. Sér. Sci. Math. Astronom. Phys. 20 (9) (1972), pp. $753-761$

[12] - Paradoxal model of Euclidean affine geometry, Bull. Acad. Polon. Sci. Sér. Sci. Math. Astron. Phys. 20 (10) (1972), pp. 845-851.

[13] L. W. Szczerba and A. Tarski, Metamathematical properties of some affine geometries, Proceedings of the 1964 International Congress for Logic, Methodology and Philosophy of 
Science. Held in Jerusalem, August 26 - September 2, 1964, North-Holland, Amsterdam, pp. $166-178$

[14] W. Szmielew, Some metamathematical problems concerning elementary hyperbolic geometry, The axiomatic method with special reference to geometry and physics, Proceedings of an The axiomatic methos wion held at the University of California, Berkeley, December 26, 1957 International Symposium held at the University of California, Berke

[15] A. Tarski, A decision method for elementary algebra and geometry, Second edition, revised, University of California Press Berkeley and Los Angeles, 1951.

[16] - What is elementary geometry? The axiomatic method with special reference to geometry and physics, Proceedings of an International Symposium held at the University of California, and phy

[17] A. Tarski, A. Mostowski and R. M. Robinson, Undecidable theories, North-Folland, Amsterdam 1953.

[18] O. Veblen, A system of axioms for geometry, Trans. Amer. Math. Soc. 5, 1904, pp. 343-384

[19] A. N. Whitehead, The axioms of descriptive geometry, Cambridge, 1907.

Accepté par la Rédaction le 12. 10. 1976

\section{Non-axiomatizability of real general affine geometry}

by

Alexander Prestel (Konstanz) and Lesław W. Szczerba (Warszawa)

Abstract. In Szczerba-Tarski [7] the question has been reised whether general affine geometry is an elementary theory of affine plane over field of real numbers restricted to non-empty, open and convex set. It is proved that the answer is negative because the first theory is given axiomatically while the second is not recursively axiomatizable.

There is a well known axiom-system of the arithmetic of real numbers containing just one non-elementary axiom, the so called continuity axiom. It follows from the considerations in Tarski [9] (see also Tarski, Mc Kinsey [11]) that by replacing the continuity axiom by the set of all of its first order instances we get an axiom system of the elementary arithmetic of real numbers. The same procedure may be carried out for real Euclidean (see Tarski [10]), hyperbolic and absolute geometry (see Szmielew [8]). In Szczerba-Tarski [6], the question was asked whether the same procedure used in the case of general affine geometry provides an axiom system for elementary real general affine geometry.

We shall prove that it is not true. In fact, elementary real general affine geometry turns out to be non-axiomatizable. Nevertheless the procedure of replacing the continuity axiom by the continuity schema provides us with an axiomatizable subtheory of elementary real general affine geometry which, in some sense, is as close as possible to it. Namely the simplest sentence discriminating these theories is of type $\forall \exists \forall$. The method of proof may be applied to the proof of some other non-axiomatizability results, e.g. we may prove by its means the non-axiomatizability of the elementary theory of rational function fields over formaly real fields, and the already known (see Rautenberg [4]) non-axiomatizability of the elementary theory of Archimedean ordered fields.

§ 1. Theorems on non-axiomatizability. Let $\mathscr{M}$ be a class of structures of the some signature (which will be regarded as fixed for the whole of this section). Let $\mathbf{T}(\mathscr{A})$ be the elementary theory of the class $\mathscr{M}$. 